\title{
Bipartite-uniform hypermaps on the sphere
}

\author{
António Breda d'Azevedo* \\ Department of Mathematics \\ University of Aveiro \\ 3810-193 Aveiro, Portugal \\ breda@mat.ua.pt
}

\author{
Rui Duarte* \\ Department of Mathematics \\ University of Aveiro \\ 3810-193 Aveiro, Portugal \\ rui@mat.ua.pt
}

Submitted: Sep 29, 2004; Accepted: Dec 7, 2006; Published: Jan 3, 2007

Mathematics Subject Classification: 05C10, 05C25, 05C30

\begin{abstract}
A hypermap is (hypervertex-) bipartite if its hypervertices can be 2-coloured in such a way that "neighbouring" hypervertices have different colours. It is bipartiteuniform if within each of the sets of hypervertices of the same colour, hyperedges and hyperfaces, all the elements have the same valency. The flags of a bipartite hypermap are naturally 2-coloured by assigning the colour of its adjacent hypervertices. A hypermap is bipartite-regular if the automorphism group acts transitively on each set of coloured flags. If the automorphism group acts transitively on the set of all flags, the hypermap is regular. In this paper we classify the bipartite-uniform hypermaps on the sphere (up to duality). Two constructions of bipartite-uniform hypermaps are given. All bipartite-uniform spherical hypermaps are shown to be constructed in this way. As a by-product we show that every bipartite-uniform hypermap $\mathcal{H}$ on the sphere is bipartite-regular. We also compute their irregularity group and index, and also their closure cover $\mathcal{H}^{\Delta}$ and covering core $\mathcal{H}_{\Delta}$.
\end{abstract}

\section{Introduction}

A map generalises to a hypermap when we remove the requirement that an edge must join two vertices at most. A hypermap $\mathcal{H}$ can be regarded as a bipartite map where one of the two monochromatic sets of vertices represent the hypervertices and the other the hyperedges of $\mathcal{H}$. In this perspective hypermaps are cellular embeddings of hypergraphs on compact connected surfaces (two-dimensional compact connected manifolds) without boundary - in this paper we deal only with the boundary-free case.

\footnotetext{
*Research partially supported by R\&DU "Matemática e Aplicações" of the University of Aveiro through "Programa Operacional Ciência, Tecnologia, Inovação" (POCTI) of the "Fundação para a Ciência e a Tecnologia" (FCT), cofinanced by the European Community fund FEDER.
} 
Usually classifications in map/hypermap theory are carried out by genus, by number of faces, by embedding of graphs, by automorphism groups or by some fixed properties such as edge-transitivity. Since Klein and Dyck [13, 11] - where certain 3-valent regular maps of genus 3 were studied in connection with constructions of automorphic functions on surfaces - most classifications of maps (and hypermaps) involve regularity or orientablyregularity (direct-regularity). The orientably-regular maps on the torus (in [10]), the orientably-regular embeddings of complete graphs (in [15]), the orientably-regular maps with automorphism groups isomorphic to $P S L(2, q)$ (in [21]) and the bicontactual regular maps (in [26]), are examples to name but a few. The just-edge-transitive maps of Jones [18] and the classification by Siran, Tucker and Watkins [22] of the edge-transitive maps on the torus, on the other hand, include another kind of "regularity" other than regularity or orientably-regularity. According to Graver and Wakins [17], an edge transitive map is determined by 14 types of automorphism groups. Among these, 11 correspond to "restricted regularity" [1]. Jones's "just-edge-transitive" maps correspond to $\Delta^{\hat{0} \hat{2}}$-regular maps of "rank 4", where $\Delta^{\hat{0} \hat{2}}$ is the normal closure of $\left\langle R_{1}, R_{0} R_{2}\right\rangle$ of index 4 in the free product $\Delta=C_{2} * C_{2} * C_{2}$ generated by the 3 reflections $R_{0}, R_{1}$ and $R_{2}$ on the sides of a hyperbolic triangle with zero internal angles; "rank 4" means that it is not $\Theta$-regular for no normal subgroup $\Theta$ of $\Delta$ of index $<4$. Moreover, the automorphism group of the toroidal edge-transitive maps realise 7 of the above 14 family-types [22]; they all correspond to restrictedly regular maps, namely of ranks 1 [the regular maps], 2 [the just-orientablyregular (or chiral) maps, the just-bipartite-regular maps, the just-face-bipartite-regular maps and the just-Petrie-bipartite-regular maps] and 4 [the just- $\Delta^{+\hat{0}}$-regular maps and the just- $\Delta^{+} \hat{2}$-regular maps] (see [1]).

In this paper we classify the "bipartite-uniform" hypermaps on the sphere. They all turn out to be "bipartite-regular". A hypermap $\mathcal{H}$ is bipartite if its hypervertices can be 2-coloured in such a way that "neighbouring" hypervertices have different colours. It is bipartite-uniform if the hypervertices of one colour, the hypervertices of the other colour, the hyperedges and the hyperfaces have common valencies $l_{1}, l_{2}, m$ and $n$ respectively. The flags of a bipartite hypermap are naturally 2-coloured by assigning the colour of their adjacent hypervertices. A bipartite hypermap is bipartite-regular if the automorphism group acts transitively on each set of coloured flags. If the automorphism group acts transitively on the whole set of flags the hypermap is regular. Bipartite-regularity corresponds to $\Delta^{\hat{0}}$-regularity [1] where $\Delta^{\hat{0}}$, a normal subgroup of index 2 in $\Delta$, is the normal closure of the subgroup generated by $R_{1}$ and $R_{2}$.

We also compute the irregularity group and the irregularity index of the bipartiteregular hypermaps $\mathcal{H}$ on the sphere as well as their closure cover $\mathcal{H}^{\Delta}$ (the smallest regular hypermap that covers $\mathcal{H}$ ) and their covering core $\mathcal{H}_{\Delta}$ (the largest regular hypermap covered by $\mathcal{H}$ ). Regular hypermaps on the sphere (see $\S 1.4$ ) are up to a $S_{3}$-duality (see $\S 1.3)$ regular maps and these are the five Platonic solids plus the two infinite families of type $(2 ; 2 ; n)$ and $(n ; n ; 1)$, and their duals. An interesting well known fact, which comes from the "universality" of the sphere, is that uniform hypermaps on the sphere are regular. According to [1] this translates to " $\Delta$-uniformity in the sphere implies $\Delta$ regularity". We may now ask for which normal subgroups $\Theta$ of finite index in $\Delta$ do 
we still have " $\Theta$-uniformity in the sphere implies $\Theta$-regularity", once the meaning of $\Theta$ uniformity is understood? As a byproduct of the classification we show in this paper that bipartite-uniformity (that is, $\Delta^{\hat{0}}$-uniformity) still implies bipartite-regularity (that is, $\Delta^{\hat{0}}$-regularity). $\Delta^{\hat{0}}$ is just one of the seven normal subgroups with index 2 in $\Delta$. The others are $\Delta^{\hat{1}}=\left\langle R_{0}, R_{2}\right\rangle^{\Delta}, \Delta^{\hat{2}}=\left\langle R_{0}, R_{1}\right\rangle^{\Delta}, \Delta^{0}=\left\langle R_{0}, R_{1} R_{2}\right\rangle^{\Delta}, \Delta^{1}=\left\langle R_{1}, R_{0} R_{2}\right\rangle^{\Delta}$, $\Delta^{2}=\left\langle R_{2}, R_{0} R_{1}\right\rangle^{\Delta}$ and $\Delta^{+}=\left\langle R_{1} R_{2}, R_{2} R_{0}\right\rangle$ (see [4] for more details). As the notation indicates they are grouped into three families, within which they differ by a dual operation. This duality says that the result is still valid if we replace $\Delta^{\hat{0}}$ by $\Delta^{\hat{1}}$ or $\Delta^{\hat{2}}$. For $\Theta=\Delta^{0}, \Delta^{1}, \Delta^{2}$, and $\Delta^{+}, \Theta$-uniformity is the same as uniformity, and since regularity implies $\Theta$-regularity, on the sphere $\Theta$-uniformity implies $\Theta$-regularity for any subgroup $\Theta$ of index 2 in $\Delta$. At the end, as a final comment, we show that on each orientable surface we can find always bipartite-chiral (that is, irregular bipartite-regular) hypermaps.

\subsection{Hypermaps}

A hypermap is combinatorially described by a four-tuple $\mathcal{H}=\left(\Omega_{\mathcal{H}} ; h_{0}, h_{1}, h_{2}\right)$ where $\Omega_{\mathcal{H}}$ is a non-empty finite set and $h_{0}, h_{1}, h_{2}$ are fixed-point free involutory permutations of $\Omega_{\mathcal{H}}$ generating a permutation group $\left\langle h_{0}, h_{1}, h_{2}\right\rangle$ acting transitively on $\Omega_{\mathcal{H}}$. The elements of $\Omega_{\mathcal{H}}$ are called flags, the permutations $h_{0}, h_{1}$ and $h_{2}$ are called canonical generators and the group $\operatorname{Mon}(\mathcal{H})=\left\langle h_{0}, h_{1}, h_{2}\right\rangle$ is the monodromy group of $\mathcal{H}$. One says that $\mathcal{H}$ is a map if $\left(h_{0} h_{2}\right)^{2}=1$. The hypervertices (or 0 -faces) of $\mathcal{H}$ correspond to $\left\langle h_{1}, h_{2}\right\rangle$-orbits on $\Omega_{\mathcal{H}}$. Likewise, the hyperedges (or 1-faces) and hyperfaces (or 2-faces) correspond to $\left\langle h_{0}, h_{2}\right\rangle$ and $\left\langle h_{0}, h_{1}\right\rangle$-orbits on $\Omega_{\mathcal{H}}$, respectively. If a flag $\omega$ belongs to the corresponding orbit determining a $k$-face $f$ we say that $\omega$ belongs to $f$, or that $f$ contains $\omega$.

We fix $\{i, j, k\}=\{0,1,2\}$. The valency of a $k$-face $f=w\left\langle h_{i}, h_{j}\right\rangle$, where $\omega \in \Omega_{\mathcal{H}}$, is the least positive integer $n$ such that $\left(h_{i} h_{j}\right)^{n} \in \operatorname{Stab}(w)$. Since $h_{i} \neq 1$ and $h_{j} \neq 1, h_{i} h_{j}$ generates a normal subgroup with index two in $\left\langle h_{i}, h_{j}\right\rangle$. It follows that $\left|\left\langle h_{i}, h_{j}\right\rangle\right|=2\left|\left\langle h_{i} h_{j}\right\rangle\right|$ and so the valency of a $k$-face is equal to half of its cardinality. $\mathcal{H}$ is uniform if its $k$-faces have the same valency $n_{k}$, for each $k \in\{0,1,2\}$. We say that $\mathcal{H}$ has type $(l ; m ; n)$ if $l, m$ and $n$ are, respectively, the least common multiples of the valencies of the hypervertices, hyperedges and hyperfaces. The characteristic of a hypermap is the Euler characteristic of its underlying surface, the imbedding surface of the underlying hypergraph (see Lemma 3 for a combinatorial definition).

A covering from a hypermap $\mathcal{H}=\left(\Omega_{\mathcal{H}} ; h_{0}, h_{1}, h_{2}\right)$ to another hypermap $\mathcal{G}=\left(\Omega_{\mathcal{G}} ; g_{0}, g_{1}\right.$, $\left.g_{2}\right)$ is a function $\psi: \Omega_{\mathcal{H}} \rightarrow \Omega_{\mathcal{G}}$ such that $h_{i} \psi=\psi g_{i}$ for all $i \in\{0,1,2\}$. The transitive action of $\operatorname{Mon}(\mathcal{G})$ on $\Omega_{\mathcal{G}}$ implies that $\psi$ is onto. By von Dyck's theorem ([16, pg 28]) the assignment $h_{i} \mapsto g_{i}$ extends to a group epimorphism $\Psi: \operatorname{Mon}(\mathcal{H}) \rightarrow \operatorname{Mon}(\mathcal{G})$ called the canonical epimorphism. The covering $\psi$ is an isomorphism if it is injective. If there exists a covering $\psi$ from $\mathcal{H}$ to $\mathcal{G}$, we say that $\mathcal{H}$ covers $\mathcal{G}$ or that $\mathcal{G}$ is covered by $\mathcal{H}$; if $\psi$ is an isomorphism we say that $\mathcal{H}$ and $\mathcal{G}$ are isomorphic and write $\mathcal{H} \cong \mathcal{G}$. An automorphism of $\mathcal{H}$ is an isomorphism $\psi: \Omega_{\mathcal{H}} \rightarrow \Omega_{\mathcal{H}}$ from $\mathcal{H}$ to itself; that is, a function $\psi$ that commutes with the canonical generators. The set of automorphisms of $\mathcal{H}$ is represented by $\operatorname{Aut}(\mathcal{H})$. As a direct consequence of the Euclidean Division Algorithm we have: 
Lemma 1. Let $\psi: \Omega_{\mathcal{H}} \rightarrow \Omega_{\mathcal{G}}$ be a covering from $\mathcal{H}$ to $\mathcal{G}$ and $\omega \in \Omega_{\mathcal{H}}$. Then the valency of the $k$-face of $\mathcal{G}$ that contains $\omega \psi$ divides the valency of the $k$-face of $\mathcal{H}$ that contains $\omega$.

Of the two groups $\operatorname{Mon}(\mathcal{H})$ and $\operatorname{Aut}(\mathcal{H})$ the first acts transitively on $\Omega=\Omega_{\mathcal{H}}$ (by definition) and the second, due to the commutativity of the automorphisms with the canonical generators, acts semi-regularly on $\Omega$; that is, the non-identity elements of $\operatorname{Aut}(\mathcal{H})$ act without fixed points. A transitive semi-regular action is called a regular action. These two actions give rise to the following inequalities:

$$
|\operatorname{Mon}(\mathcal{H})| \geq|\Omega| \geq|\operatorname{Aut}(\mathcal{H})| .
$$

Moreover, each of the above equalities implies the other. An equality in the first of these inequalities implies that $\operatorname{Mon}(\mathcal{H})$ acts semi-regularly (hence regularly) on $\Omega$, while an equality on the second implies that $\operatorname{Aut}(\mathcal{H})$ acts transitively (hence regularly) on $\Omega$. If $\operatorname{Mon}(\mathcal{H})$ acts regularly on $\Omega$, or equivalently if $\operatorname{Aut}(\mathcal{H})$ acts regularly on $\Omega$, the hypermap $\mathcal{H}$ is regular.

Each hypermap $\mathcal{H}$ gives rise to a permutation representation $\rho_{\mathcal{H}}: \Delta \rightarrow \operatorname{Mon}(\mathcal{H})$, $R_{i} \mapsto h_{i}$, where $\Delta$ is the free product $C_{2} * C_{2} * C_{2}$ with presentation $\Delta=\left\langle R_{0}, R_{1}, R_{2}\right|$ $\left.R_{0}{ }^{2}=R_{1}{ }^{2}=R_{2}{ }^{2}=1\right\rangle$. The group $\Delta$ acts naturally and transitively on $\Omega_{\mathcal{H}}$ via $\rho_{\mathcal{H}}$. The stabiliser $H=\operatorname{Stab}_{\Delta}(\omega)$ of a flag $\omega \in \Omega_{\mathcal{H}}$ under the action of $\Delta$ is called the hypermap subgroup of $\mathcal{H}$; this is unique up to conjugation in $\Delta$. The valency of a $k$-face containing $\omega$ is the least positive integer $n$ such that $\left(R_{i} R_{j}\right)^{n} \in H$; more generally, the valency of a $k$-face containing the flag $\sigma=\omega \cdot g=\omega(g) \rho_{\mathcal{H}} \in \Omega_{\mathcal{H}}$, where $g \in \Delta$, is the least positive integer $n$ such that $\left(R_{i} R_{j}\right)^{n} \in \operatorname{Stab}_{\Delta}(\sigma)=\operatorname{Stab}_{\Delta}(\omega \cdot g)=\operatorname{Stab}_{\Delta}(\omega)^{g}=H^{g}$.

Denote by $\operatorname{Alg}(\mathcal{H})=\left(\Delta /{ }_{r} H ; a_{0}, a_{1}, a_{2}\right)$ where $a_{i}: \Delta /{ }_{r} H \rightarrow \Delta /{ }_{r} H, H g \mapsto H g H_{\Delta} R_{i}=$ $H g R_{i}$. It is easy to see that $\operatorname{Alg}(\mathcal{H}) \cong \mathcal{H}$. We say that $\operatorname{Alg}(\mathcal{H})$ is the algebraic presentation of $\mathcal{H}$. Moreover, it is well known that:

1. A hypermap $\mathcal{H}$ is regular if and only if its hypermap subgroup $H$ is normal in $\Delta$.

2. A regular hypermap is necessarily uniform.

Since $\operatorname{Alg}(\mathcal{H})$ and $\mathcal{H}$ are isomorphic, we will not differentiate one from the other.

Following [1], if $H<\Theta$ for a given $\Theta \triangleleft \Delta$, we say that $\mathcal{H}$ is $\Theta$-conservative. A $\Delta^{+}$-conservative hypermap is better known as an orientable hypermap. An automorphism of an orientable hypermap either preserves the two $\Delta^{+}$-orbits or permutes them. Those that preserve $\Delta^{+}$-orbits are called orientation-preserving automorphisms. The set of orientation-preserving automorphisms is a subgroup of $\operatorname{Aut}(\mathcal{H})$ and is denoted by $\operatorname{Aut}^{+}(\mathcal{H})$. If $\mathcal{H}$ is $\Delta^{\hat{0}}$-conservative (resp. $\Delta^{\hat{1}}$-conservative, resp. $\Delta^{\hat{2}}$-conservative) we say that $\mathcal{H}$ is bipartite, vertex-bipartite or 0-bipartite (resp. edge-bipartite or 1-bipartite, resp. face-bipartite or 2-bipartite).

Lemma 2. If $\mathcal{H}$ is bipartite and $\omega \in \Omega_{\mathcal{H}}$, then the valencies of the hyperedge and the hyperface that contain $\omega$ must be even. 
Proof. If $m$ and $n$ are the valencies of the hyperedge and the hyperface that contain $\omega=H d, d \in \Delta$, then $\left(R_{2} R_{0}\right)^{m},\left(R_{0} R_{1}\right)^{n} \in H^{d} \subseteq \Delta^{\hat{0}}$. Therefore $m$ and $n$ must be even.

If $H \triangleleft \Delta^{+}$, we say that $\mathcal{H}$ is orientably-regular. If $H \triangleleft \Delta^{\hat{0}}$ (resp. $H \triangleleft \Delta^{\hat{1}}$ and $H \triangleleft \Delta^{\hat{2}}$ ), we say that $\mathcal{H}$ is vertex-bipartite-regular (resp. edge-bipartite-regular and facebipartite-regular). If $\mathcal{H}$ is vertex-bipartite-regular (resp. edge-bipartite-regular, resp. face-bipartite-regular) but not regular, we say that $\mathcal{H}$ is vertex-bipartite-chiral (resp. edgebipartite-chiral, resp. face-bipartite-chiral). We will use bipartite-regular and bipartitechiral in place of vertex-bipartite-regular and vertex-bipartite-chiral for short.

A bipartite-uniform hypermap is a bipartite hypermap such that all the hypervertices in the same $\Delta^{\hat{0}}$-orbit have the same valency, as do all the hyperedges and all the hyperfaces. The bipartite-type of a bipartite-uniform hypermap $\mathcal{H}$ is a four-tuple $\left(l_{1}, l_{2} ; m ; n\right.$ ) (or $\left.\left(l_{2}, l_{1} ; m ; n\right)\right)$ where $l_{1}$ and $l_{2}\left(l_{1} \leq l_{2}\right)$ are the valencies (not necessarily distinct) of the hypervertices of $\mathcal{H}, m$ is the valency of the hyperedges of $\mathcal{H}$ and $n$ is the valency of the hyperfaces of $\mathcal{H}$. We note that if $\mathcal{H}$ is a bipartite-uniform hypermap of bipartite-type $\left(l_{1}, l_{2} ; m ; n\right)$, then $m$ and $n$ must be even by Lemma 2 .

\subsection{Euler formula for uniform hypermaps}

Using the well known Euler formula for maps one easily gets the following well known result:

Lemma 3 (Euler formula for hypermaps). Let $\mathcal{H}$ be a hypermap with $V$ hypervertices, $E$ hyperedges and $F$ hyperfaces. If $\mathcal{H}$ has underlying surface $\mathcal{S}$ with Euler characteristic $\chi$, then $\chi=V+E+F-\frac{\left|\Omega_{\mathcal{H}}\right|}{2}$. (See for example [28] and the references therein.)

If $\mathcal{H}$ is uniform of type $(l, m, n)$, then $V=\frac{\left|\Omega_{\mathcal{H}}\right|}{2 l}, E=\frac{\left|\Omega_{\mathcal{H}}\right|}{2 m}$ and $F=\frac{\left|\Omega_{\mathcal{H}}\right|}{2 n}$. Replacing the values of $V, E$ and $F$ in the last formula, we get:

Corollary 4 (Euler formula for uniform hypermaps).

$$
\chi=\frac{\left|\Omega_{\mathcal{H}}\right|}{2}\left(\frac{1}{l}+\frac{1}{m}+\frac{1}{n}-1\right) .
$$

\subsection{Duality}

A non-inner automorphism $\psi$ of $\Delta$ (that is, an automorphism not arising from a conjugation) gives rise to an operation on hypermaps by transforming a hypermap $\mathcal{H}=$ $\left(\Delta_{/ r} H, H_{\Delta} R_{0}, H_{\Delta} R_{1}, H_{\Delta} R_{2}\right)$, with hypermap-subgroup $H$, into its operation-dual

$$
\begin{aligned}
D_{\psi}(\mathcal{H}) & =\left(\Delta /_{r} H \psi ;(H \psi)_{\Delta} R_{0},(H \psi)_{\Delta} R_{1},(H \psi)_{\Delta} R_{2}\right) \\
& =\left(\Delta /_{r} H \psi ; H_{\Delta} \psi R_{0}, H_{\Delta} \psi R_{1}, H_{\Delta} \psi R_{2}\right)
\end{aligned}
$$

with hypermap-subgroup $H \psi$ (see $[14,19,20]$ for more details). Note that if $\psi$ is inner, then $D_{\psi}(\mathcal{H})$ is isomorphic to $\mathcal{H}$. In particular, each permutation $\sigma \in S_{\{0,1,2\}} \backslash\{i d\}$ induces 
a non-inner automorphism $\sigma^{\circ}: \Delta \longrightarrow \Delta$ by assigning $R_{i} \mapsto R_{i \sigma}$, for $i=0,1,2$. This automorphism induces an operation $\mathcal{D}_{\sigma}$ on hypermaps by assigning the hypermap-subgroup $H$ of $\mathcal{H}$ to a hypermap-subgroup $H \sigma^{\circ}$. Such an operator transforms each hypermap $\mathcal{H}=\left(\Omega_{\mathcal{H}} ; h_{0}, h_{1}, h_{2}\right)$ into its $\sigma$-dual $D_{\sigma}(\mathcal{H}) \cong\left(\Omega_{\mathcal{H}} ; h_{0 \sigma^{-1}}, h_{1 \sigma^{-1}}, h_{2 \sigma^{-1}}\right)$. We note that the $k$-faces of $\mathcal{H}$ are the $k \sigma$-faces of $\mathcal{D}_{\sigma}(\mathcal{H})$. From this note and the definition of $\sigma$-duality one easily get the following properties of $\mathcal{D}_{\sigma}$.

Lemma 5 (Properties of $\mathcal{D}_{\sigma}$ ). Let $\mathcal{H}, \mathcal{G}$ be two hypermaps and $\sigma, \tau \in S_{\{0,1,2\}}$. Then (1) $\mathcal{D}_{1}(\mathcal{H})=\mathcal{H}$, where $1=i d \in S_{\{0,1,2\}} ; \quad$ (2) $\mathcal{D}_{\tau}\left(\mathcal{D}_{\sigma}(\mathcal{H})\right)=\mathcal{D}_{\sigma \tau}(\mathcal{H})$; (3) If $\mathcal{H}$ covers $\mathcal{G}$, then $\mathcal{D}_{\sigma}(\mathcal{H})$ covers $\mathcal{D}_{\sigma}(\mathcal{G})$; (4) If $\mathcal{H} \cong \mathcal{G}$, then $\mathcal{D}_{\sigma}(\mathcal{H}) \cong \mathcal{D}_{\sigma}(\mathcal{G})$; (5) If $\mathcal{H}$ is uniform, then $\mathcal{D}_{\sigma}(\mathcal{H})$ is uniform; (6) If $\mathcal{H}$ is k-bipartite-uniform, then $\mathcal{D}_{\sigma}(\mathcal{H})$ is k $\sigma$-bipartite-uniform; (7) If $\mathcal{H}$ is regular, then $\mathcal{D}_{\sigma}(\mathcal{H})$ is regular; (8) If $\mathcal{H}$ is $k$-bipartite-regular, then $\mathcal{D}_{\sigma}(\mathcal{H})$

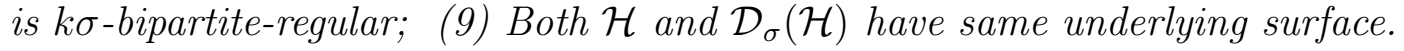

\subsection{Spherical uniform hypermaps}

A hypermap $\mathcal{H}$ is spherical if its underlying surface is a sphere (i.e if its Euler characteristic is 2). By taking $l \leq m \leq n$ and $\chi=2$ in the Euler formula one easily sees that $l<3$. A simple analysis to the above inequality leads us to the following table of possible types (up to duality):

\begin{tabular}{ccc|ccc|ccc|c}
$l$ & $m$ & $n$ & $V$ & $E$ & $F$ & $\left|\Omega_{\mathcal{H}}\right|$ & $\operatorname{Mon}(\mathcal{H})$ & $\mathcal{H}$ & Aut $^{+}(\mathcal{H})$ \\
\hline 1 & $k$ & $k$ & $k$ & 1 & 1 & $2 k$ & $D_{k}$ & $\mathcal{D}_{(02)}\left(\mathcal{D}_{k}\right)$ & $C_{k}$ \\
2 & 2 & $k$ & $k$ & $k$ & 2 & $4 k$ & $D_{k} \times C_{2}$ & $\mathcal{P}_{k}$ & $C_{k}$ \\
2 & 3 & 3 & 6 & 4 & 4 & 24 & $S_{4}$ & $\mathcal{D}_{(01)}(\mathcal{T})$ & $A_{4}$ \\
2 & 3 & 4 & 12 & 8 & 6 & 48 & $S_{4} \times C_{2}$ & $\mathcal{D}_{(01)}(\mathcal{C})$ & $S_{4}$ \\
2 & 3 & 5 & 30 & 20 & 12 & 120 & $A_{5} \times C_{2}$ & $\mathcal{D}_{(01)}(\mathcal{D})$ & $A_{5}$ \\
\hline
\end{tabular}

Table 1: Possible values (up to duality) for type $(l ; m ; n)$.

Lemma 6. All uniform hypermaps on the sphere are regular.

This result arises because each type $(l ; m ; n)$ in Table 1 determines a cocompact subgroup $H=\left\langle\left(R_{1} R_{2}\right)^{l},\left(R_{2} R_{0}\right)^{m},\left(R_{0} R_{1}\right)^{n}\right\rangle^{\Delta}$ with index $\left|\Omega_{\mathcal{H}}\right|$ in the free product $\Delta=$ $C_{2} * C_{2} * C_{2}$ generated by $R_{0}, R_{1}$ and $R_{2}$.

Let $\mathcal{T}, \mathcal{C}, \mathcal{O}, \mathcal{D}$ and $\mathcal{I}$ denote the 2-skeletons of the tetrahedron, the cube, the octahedron, the dodecahedron and the icosahedron. These are, up to isomorphism, the unique uniform hypermaps of type $(3 ; 2 ; 3),(3 ; 2 ; 4),(4 ; 2 ; 3),(3 ; 2 ; 5)$ and $(5 ; 2 ; 3)$ respectively, on the sphere; note that $\mathcal{O} \cong \mathcal{D}_{(02)}(\mathcal{C})$ and $\mathcal{I} \cong \mathcal{D}_{(02)}(\mathcal{D})$. Together with the infinite families of hypermaps $\mathcal{D}_{n}$ with monodromy group $D_{n}$ and $\mathcal{P}_{n}$ with monodromy group $D_{n} \times C_{2}$ $(n \in \mathbb{N})$, of types $(n ; n ; 1)$ and $(2 ; 2 ; n)$, respectively, they complete, up to duality and isomorphism, the uniform spherical hypermaps. 

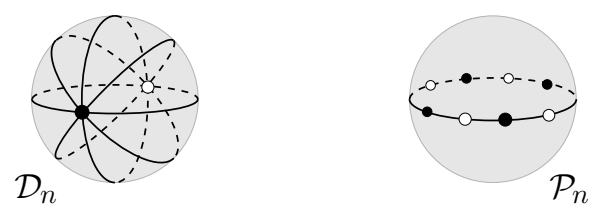

The last column of Table 1 displays the uniform spherical hypermaps (which are regular by last lemma) of type $(l ; m ; n)$ with $l \leq m \leq n$.

Lemma 7. If $\mathcal{H}$ is a hypermap such that all hyperfaces have valency 1 , then $\mathcal{H}$ is the "dihedral" hypermap $\mathcal{D}_{n}$, a regular hypermap on the sphere with $n$ hyperfaces.

Proof. Let $H$ be a hypermap-subgroup of $\mathcal{H}$. All hyperfaces having valency 1 implies that $R_{0} R_{1} \in H^{d}$ for all $d \in \Delta$ (i.e., $R_{0} R_{1}$ stabilises all the flags). Then $H\left\langle R_{1}, R_{2}\right\rangle=$ $H\left\langle R_{0}, R_{2}\right\rangle=H\left\langle R_{0}, R_{1}, R_{2}\right\rangle=\Delta /{ }_{r} H=\Omega$; that is, $\mathcal{H}$ has only one hypervertex and one hyperedge. Hence $\mathcal{H} \cong \mathcal{D}_{n}$, where $n$ is the valency of the hyperedge and the hyperface of $\mathcal{H}$.

\section{Constructing bipartite hypermaps}

By the Reidemeister-Schreier rewriting process [16] it can be shown that

$$
\Delta^{\hat{0}} \cong C_{2} * C_{2} * C_{2} * C_{2}=\left\langle R_{1}\right\rangle *\left\langle R_{2}\right\rangle *\left\langle R_{1}^{R_{0}}\right\rangle *\left\langle R_{2}^{R_{0}}\right\rangle \text {. }
$$

As a consequence we have an epimorphism $\varphi: \Delta^{\hat{0}} \longrightarrow \Delta$.

Any such epimorphism $\varphi$ induces a transformation (not an operation) of hypermaps, by transforming each hypermap $\mathcal{H}=\left(\Omega_{\mathcal{H}} ; h_{0}, h_{1}, h_{2}\right)$ with hypermap subgroup $H$ into a hypermap $\mathcal{H}^{\varphi^{-1}}=\left(\Omega ; t_{0}, t_{1}, t_{2}\right)$ with hypermap subgroup $H \varphi^{-1}$.

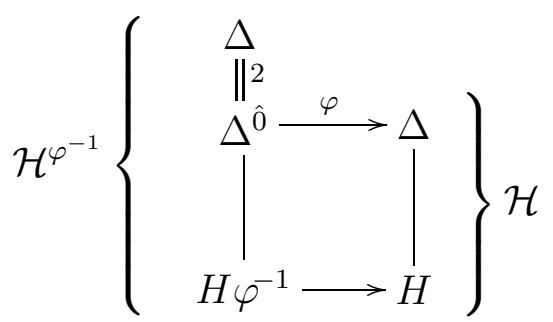

Algebraically, $\mathcal{H}^{\varphi^{-1}}=\left(\Delta / r H \varphi^{-1} ; s_{0}, s_{1}, s_{2}\right)$ with $s_{i}=\left(H \varphi^{-1}\right)_{\Delta} R_{i}$ acting on $\Omega=\Delta / r H \varphi^{-1}$ by right multiplication. Here $\left(H \varphi^{-1}\right)_{\Delta}$ denotes the core of $H \varphi^{-1}$ in $\Delta$. In the following lemma we list three elementary, but useful, properties of this transformation $\varphi$.

Lemma 8. Let $g \in \Delta, W=\left(H \varphi^{-1}\right)_{\Delta} w \in \Delta /\left(H \varphi^{-1}\right)_{\Delta}=\operatorname{Mon}\left(\mathcal{H}^{\varphi^{-1}}\right)$ and $H \varphi^{-1} g \in \Omega$ be a flag of $\mathcal{H}^{\varphi^{-1}}$. Then,

(1) If $g \in \Delta^{\hat{0}}$, then $\left(H \varphi^{-1}\right)^{g}=H^{g \varphi} \varphi^{-1}$. If $g \notin \Delta^{\hat{0}}$, then $\left(H \varphi^{-1}\right)^{g}=\left(H^{\left(g R_{0}\right) \varphi} \varphi^{-1}\right)^{R_{0}}$. 
(2) $\left(H \varphi^{-1}\right)_{\Delta^{\hat{0}}}=H_{\Delta} \varphi^{-1}$ and $\left(H \varphi^{-1}\right)_{\Delta}=H_{\Delta} \varphi^{-1} \cap\left(H_{\Delta} \varphi^{-1}\right)^{R_{0}}$.

(3) $W \in \operatorname{Stab}\left(H \varphi^{-1} g\right) \Leftrightarrow w \in\left(H \varphi^{-1}\right)^{g} \Leftrightarrow\left\{\begin{array}{ll}w \varphi \in H^{g \varphi}, & \text { if } g \in \Delta^{\hat{0}} \\ w^{R_{0}} \varphi \in H^{\left(g R_{0}\right) \varphi}, & \text { if } g \notin \Delta^{\hat{0}} .\end{array}\right.$ Moreover, $W \in \operatorname{Stab}\left(H \varphi^{-1} g\right)$ implies that $w \in \Delta^{\hat{0}}$.

Proof. (1) If $g \in \Delta^{\hat{0}}$, then $x \in H^{g \varphi} \varphi^{-1} \Leftrightarrow x \varphi \in H^{g \varphi} \Leftrightarrow(x \varphi)^{(g \varphi)^{-1}}=(x \varphi)^{g^{-1} \varphi}=x^{g^{-1}} \varphi \in$ $H \Leftrightarrow x \in\left(H \varphi^{-1}\right)^{g}$. If $g \notin \Delta^{\hat{0}}$, then $g R_{0} \in \Delta^{\hat{0}}$ and so $\left(H \varphi^{-1}\right)^{g}=\left(\left(H \varphi^{-1}\right)^{\left(g R_{0}\right)}\right)^{R_{0}}=$ $\left(H^{\left(g R_{0}\right) \varphi} \varphi^{-1}\right)^{R_{0}}$.

(2) Since $\varphi$ is onto, the above item translates into these two results.

(3) $W \in \operatorname{Stab}\left(H \varphi^{-1} g\right)=\operatorname{Stab}\left(H \varphi^{-1}\right)^{g} \Leftrightarrow w \in\left(H \varphi^{-1}\right)^{g}$. Since $H \varphi^{-1} \triangleleft \Delta^{\hat{0}}$, this implies that $w \in \Delta^{\hat{0}}$.

If $g \in \Delta^{\hat{0}}$, then $w \in\left(H \varphi^{-1}\right)^{g} \stackrel{(1)}{=} H^{g \varphi} \varphi^{-1} \Leftrightarrow w \varphi \in H^{g \varphi}$.

If $g \notin \Delta^{\hat{0}}$, then $g R_{0} \in \Delta^{\hat{0}}$ and so, by above, $w \in\left(H \varphi^{-1}\right)^{g} \Leftrightarrow w^{R_{0}} \in\left(H \varphi^{-1}\right)^{g R_{0}} \Leftrightarrow$ $\left(w^{R_{0}}\right) \varphi \in H^{\left(g R_{0}\right) \varphi}$.

Remark: For simplicity we will not distinguish $W$ from $w$, and so we will see $W$ as a word on $R_{0}, R_{1}$ and $R_{2}$ in $\Delta$ instead of a coset word $\left(H \varphi^{-1}\right){ }_{\Delta} w$.

Theorem 9. If $\mathcal{H} \cong \mathcal{G}^{\varphi^{-1}}$ for some hypermap $\mathcal{G}$, then $\Delta^{\hat{0}}$-Mon $(\mathcal{H}) \cong \operatorname{Mon}(\mathcal{G})$.

Proof. By Lemma 8(2) we deduce that

$\Delta^{\hat{0}}-\operatorname{Mon}(\mathcal{H})=\Delta^{\hat{0}} / H_{\Delta^{\hat{0}}}=\Delta^{\hat{0}} /\left(G \varphi^{-1}\right)_{\Delta^{\hat{0}}}=\Delta^{\hat{0}} / G_{\Delta} \varphi^{-1} \cong \Delta / G_{\Delta}=\operatorname{Mon}(\mathcal{G})$.

Among many possible canonical epimorphisms $\varphi: \Delta^{\hat{0}} \rightarrow \Delta$, there are two that induce transformations preserving the underlying surface, namely $\varphi_{W}$ and $\varphi_{P}$ defined by

$$
\begin{array}{cccc}
R_{1} \varphi_{W}=R_{1}, & R_{2} \varphi_{W}=R_{2}, & R_{1}{ }^{R_{0}} \varphi_{W}=R_{0}, & R_{2}{ }^{R_{0}} \varphi_{W}=R_{2}, \\
R_{1} \varphi_{P}=R_{1}, & R_{2} \varphi_{P}=R_{2}, & R_{1}{ }^{R_{0}} \varphi_{P}=R_{0}, & R_{2}{ }^{R_{0}} \varphi_{P}=R_{0} .
\end{array}
$$

Denote by $\operatorname{Wal}(\mathcal{H})$ the hypermap $\mathcal{H}^{\varphi_{W}{ }^{-1}}$ and by $\operatorname{Pin}(\mathcal{H})$ the hypermap $\mathcal{H}^{\varphi_{P}^{-1}}$. $\operatorname{Wal}(\mathcal{H})$ is a map; in fact, since $\left(R_{0} R_{2}\right)^{2}=R_{2}{ }^{R_{0}} R_{2}$ and $\left(\left(R_{0} R_{2}\right)^{2}\right)^{R_{0}}=R_{2} R_{2} R_{0}$ we have $\left(R_{0} R_{2}\right)^{2} \varphi_{W}=$ $\left(\left(R_{0} R_{2}\right)^{2}\right)^{R_{0}} \varphi_{W}=1$, and hence, by Lemma 8(3), for all $g \in \Delta,\left(R_{0} R_{2}\right)^{2} \in \operatorname{Stab}\left(H \varphi_{W}^{-1} g\right)$.

Both hypermaps $W a l(\mathcal{H})$ and $\operatorname{Pin}(\mathcal{H})$ have the same underlying surface as $\mathcal{H}$ but while $W a l(\mathcal{H})$ is a map (bipartite map since $H \varphi_{W}^{-1} \subseteq \Delta^{\hat{0}}$ ), the well known Walsh bipartite map of $\mathcal{H}[24,4], \operatorname{Pin}(\mathcal{H})$ is not necessarily a map. 


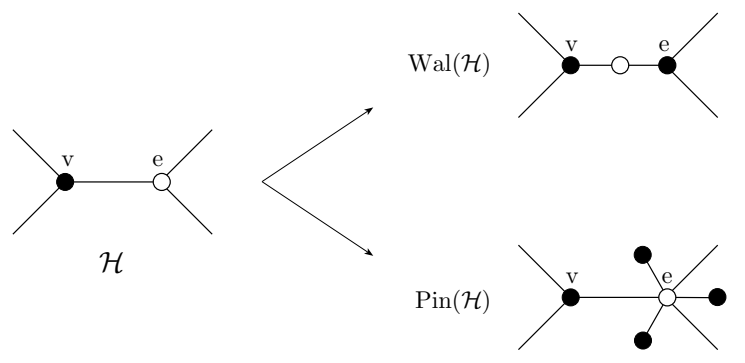

Figure 1: Topological construction of $\operatorname{Wal}(\mathcal{H})$ and $\operatorname{Pin}(\mathcal{H})$.

Theorem 10 (Properties of $\varphi_{W}$ ). Let $\mathcal{H}$ be a hypermap. Then:

1. $\mathcal{H}$ is uniform of type $(l ; m ; n)$ if and only if $W$ al $(\mathcal{H})$ is bipartite-uniform of bipartitetype $(l, m ; 2 ; 2 n)$ if $l \leq m$ or $(m, l ; 2 ; 2 n)$ if $l \geq m$;

2. $\mathcal{H}$ is regular if and only if $W a l(\mathcal{H})$ is bipartite-regular.

Proof. Let $H$ be a hypermap subgroup of $\mathcal{H}$. Then $H \varphi_{W}^{-1}$ is a hypermap subgroup of $\operatorname{Wal}(\mathcal{H})$.

$(10.1)(\Rightarrow)$ Let us suppose that $\mathcal{H}$ is uniform of type $(l ; m ; n)$. Note first that

$$
\begin{aligned}
& R_{1} R_{2}=\left(R_{1} R_{2}\right) \varphi_{W}, \\
& R_{0} R_{2}=\left(R_{1}^{R_{0}} R_{2}{ }^{R_{0}}\right) \varphi_{W}=\left(R_{1} R_{2}\right)^{R_{0}} \varphi_{W}, \\
& R_{0} R_{1}=\left(R_{1}{ }^{R_{0}} R_{1}\right) \varphi_{W}=\left(R_{0} R_{1}\right)^{2} \varphi_{W} .
\end{aligned}
$$

Let $W$ denote a word in $R_{0}, R_{1}, R_{2}$ and $\omega g \in \Omega_{W a l(\mathcal{H})}$ be any flag $(g \in \Delta)$. We already know that the valency of the hyperedge containing $\omega g$ is $2(W a l(\mathcal{H})$ is a map) and that the valency of the hyperface contains $\omega g$ is even. Let $l^{\prime}$ and $n^{\prime}$ be the valencies of the hypervertex and the hyperface containing $\omega g$, respectively.

(1) $g \in \Delta^{\hat{0}}$. From (1) and Lemma 8(1) we have $\left(R_{1} R_{2}\right)^{k} \in H^{g \varphi_{W}}$ if and only if $\left(R_{1} R_{2}\right)^{k} \in$ $H^{g \varphi_{W}} \varphi_{W}^{-1}=\left(H \varphi_{W}^{-1}\right)^{g}$; that is, according to Lemma 8(3),

$$
\left(R_{1} R_{2}\right)^{k} \in \operatorname{Stab}\left(H\left(g \varphi_{W}\right)\right) \Leftrightarrow\left(R_{1} R_{2}\right)^{k} \in \operatorname{Stab}\left(\left(H \varphi_{W}^{-1}\right) g\right) .
$$

Analogously, from (3) we get $\left(R_{0} R_{1}\right)^{k} \in H^{g \varphi_{W}}$ if and only if $\left(R_{0} R_{1}\right)^{2 k} \in H^{g \varphi_{W}} \varphi_{W}^{-1}=$ $\left(H \varphi_{W}^{-1}\right)^{g}$ that is, according to Lemma $8(3)$,

$$
\left(R_{0} R_{1}\right)^{k} \in \operatorname{Stab}\left(H\left(g \varphi_{W}\right)\right) \Leftrightarrow\left(R_{0} R_{1}\right)^{2 k} \in \operatorname{Stab}\left(\left(H \varphi_{W}^{-1}\right) g\right) .
$$

Now the uniformity of $\mathcal{H}$ implies $l^{\prime}=l$ and $n^{\prime}=2 n$.

(2) $g \notin \Delta^{\hat{0}}$. Since $g R_{0} \in \Delta^{\hat{0}}$ we get from (2),

$$
\begin{aligned}
\left(R_{0} R_{2}\right)^{k} \in H^{\left(g R_{0}\right) \varphi_{W}} & \Leftrightarrow\left(\left(R_{1} R_{2}\right)^{R_{0}}\right)^{k} \in H^{\left(g R_{0}\right) \varphi_{W}} \varphi_{W}^{-1}=\left(H \varphi_{W}^{-1}\right)^{g R_{0}} \\
& \Leftrightarrow\left(R_{1} R_{2}\right)^{k} \in\left(H \varphi_{W}^{-1}\right)^{g} ;
\end{aligned}
$$


and from (3),

$$
\begin{aligned}
\left(R_{0} R_{1}\right)^{k} \in H^{\left(g R_{0}\right) \varphi_{W}} & \Leftrightarrow\left(R_{0} R_{1}\right)^{2 k} \in H^{g R_{0} \varphi_{W}} \varphi_{W}^{-1}=\left(H \varphi_{W}^{-1}\right)^{g R_{0}} \\
& \Leftrightarrow\left(R_{1} R_{0}\right)^{2 k} \in\left(H \varphi_{W}^{-1}\right)^{g} .
\end{aligned}
$$

This implies that

$$
\begin{aligned}
& \left(R_{0} R_{2}\right)^{k} \in \operatorname{Stab}\left(H\left(g R_{0}\right) \varphi_{W}\right) \Leftrightarrow\left(R_{1} R_{2}\right)^{k} \in \operatorname{Stab}\left(H \varphi_{W}^{-1} g\right), \\
& \left(R_{0} R_{1}\right)^{k} \in \operatorname{Stab}\left(H\left(g R_{0}\right) \varphi_{W}\right) \Leftrightarrow\left(R_{1} R_{0}\right)^{2 k} \in \operatorname{Stab}\left(H \varphi_{W}^{-1} g\right) .
\end{aligned}
$$

Likewise, the uniformity of $\mathcal{H}$ now implies that $l^{\prime}=m$ and $n^{\prime}=2 n$.

Combining (1) and (2) and assuming, without loss of generality, that $l \leq m$, we find that $\operatorname{Wal}(\mathcal{H})$ is bipartite-uniform of bipartite-type $(l, m ; 2 ; 2 n)$.

$(\Leftarrow)$ Let us assume that $\operatorname{Wal}(\mathcal{H})$ is bipartite-uniform of bipartite-type $(l, m ; 2 ; 2 n)$. Being bipartite, $\operatorname{Wal}(\mathcal{H})$ has two orbits of vertices: the "black" vertices, all with valency $l$ (say), and the "white" vertices, all with valency $m$. Without loss of generality, all the flags $H \varphi_{W}^{-1} g, g \in \Delta^{\hat{0}}$, are adjacent to "black" vertices while all the flags $H \varphi_{W}^{-1} g R_{0}$, $g \in \Delta^{\hat{0}}$, are adjacent to "white" vertices. As seen before, the equivalence (1) for $g \in \Delta^{\hat{0}}$ gives rise to the equivalence (4), which expresses the fact that all the hypervertices of $\mathcal{H}$ have the same valency $l$; the equivalence (2) for $g \notin \Delta^{\hat{0}}$ gives rise to the equivalence (6), which says that all the hyperedges of $\mathcal{H}$ have the same valency $m$; finally, the equivalence (3) gives rise to the equivalence (5) if $g \in \Delta^{\hat{0}}$ or the equivalence (7) if $g \notin \Delta^{\hat{0}}$, and they express the fact that all the hyperfaces of $\mathcal{H}$ have the same valency $n$. Hence $\mathcal{H}$ is uniform of type $(l ; m ; n)$ (or $(m ; l ; n)$ since the positional order of $l$ and $m$ in the bipartite-type of $\operatorname{Wal}(\mathcal{H})$ is ordered by increasing value).

(10.2) $\mathcal{H}$ is regular $\Leftrightarrow H \triangleleft \Delta \Leftrightarrow H \varphi_{W}^{-1} \triangleleft \Delta^{\hat{0}} \Leftrightarrow W a l(\mathcal{H})$ is bipartite-regular since $\varphi_{W}$ is an epimorphism.

Theorem 11. $\mathcal{H}$ is a bipartite map if and only if $\mathcal{H} \cong W$ al $(\mathcal{G})$ for some hypermap $\mathcal{G}$.

Proof. Only the necessary condition needs to be proved. If $\mathcal{H}$ is a bipartite map, then $H \subseteq$ $\Delta^{\hat{0}}$. Since $\mathcal{H}$ is a map, $\left(\left(R_{0} R_{2}\right)^{2}\right)^{g} \in H$ for all $g \in \Delta$; therefore $\operatorname{ker} \varphi_{W}=\left\langle\left(R_{0} R_{2}\right)^{2}\right\rangle^{\Delta^{\hat{0}}} \subseteq H$. This implies that $H \varphi_{W} \varphi_{W}^{-1}=H \operatorname{ker} \varphi_{W}=H$ and hence $\mathcal{H} \cong \operatorname{Wal}(\mathcal{G})$ where $\mathcal{G}$ is a hypermap with hypermap subgroup $G=H \varphi_{W}$.

Theorem 12 (Properties of $\left.\varphi_{P}\right)$. Let $\mathcal{H}$ be a hypermap. Then,

1. $\operatorname{Pin}(\mathcal{H})$ is a bipartite hypermap such that all hypervertices in one $\Delta^{0}$-orbit have valency 1 ;

2. $\mathcal{H}$ is uniform of type $(l ; m ; n)$ if and only if $\operatorname{Pin}(\mathcal{H})$ is bipartite-uniform of bipartitetype $(1, l ; 2 m ; 2 n)$;

3. $\mathcal{H}$ is regular if and only if $\operatorname{Pin}(\mathcal{H})$ is bipartite-regular. 
Proof. Let $H$ be a hypermap subgroup of $\mathcal{H}$. Then $H \varphi_{P}^{-1}$ is a hypermap subgroup of $\operatorname{Pin}(\mathcal{H})$.

(1) $\operatorname{Pin}(\mathcal{H})$ is bipartite since $H \varphi_{P}^{-1} \subseteq \Delta^{\hat{0}}$. We have $\left(R_{1} R_{2}\right)^{R_{0}} \varphi_{P}=\left(R_{1}{ }^{R_{0}} R_{2}{ }^{R_{0}}\right) \varphi_{P}=1$; therefore, by Lemma $2(2), R_{1} R_{2} \in \operatorname{Stab}\left(H \varphi_{P}^{-1} g\right)$ for all $g \notin \Delta^{\hat{0}}$, i.e, all hypervertices in the same $\Delta^{\hat{0}}$-orbit of the hypervertex containing the flag $H \varphi_{P}^{-1} R_{0}$ have valency 1.

(2) Let us suppose that $\mathcal{H}$ is uniform of type $(l ; m ; n)$. We proceed similarly as for $\varphi_{W}$, keeping in mind that all hypervertices of $\operatorname{Pin}(\mathcal{H})$ adjacent to flags $H \varphi_{P}^{-1} g$, for $g \notin \Delta^{\hat{0}}$, have valency 1 . Starting from the equalities,

$$
\begin{aligned}
& R_{1} R_{2}=\left(R_{1} R_{2}\right) \varphi_{P}, \\
& R_{0} R_{2}=\left(R_{2}{ }^{R_{0}} R_{2}\right) \varphi_{P}=\left(R_{0} R_{2}\right)^{2} \varphi_{P}, \\
& R_{0} R_{1}=\left(R_{1}{ }^{R_{0}} R_{1}\right) \varphi_{P}=\left(R_{0} R_{1}\right)^{2} \varphi_{P} .
\end{aligned}
$$

one gets the following equivalences,

$$
\begin{aligned}
& \left(R_{1} R_{2}\right)^{k} \in \operatorname{Stab}\left(H g \varphi_{P}\right) \Leftrightarrow\left(R_{1} R_{2}\right)^{k} \in \operatorname{Stab}\left(H \varphi_{P}^{-1} g\right), \forall g \in \Delta^{\hat{0}}, \\
& \left(R_{0} R_{2}\right)^{k} \in \operatorname{Stab}\left(H g \varphi_{P}\right) \Leftrightarrow\left(R_{0} R_{2}\right)^{2 k} \in \operatorname{Stab}\left(H \varphi_{P}^{-1} g\right), \forall g \in \Delta^{\hat{0}}, \\
& \left(R_{0} R_{2}\right)^{k} \in \operatorname{Stab}\left(H\left(g R_{0}\right) \varphi_{P}\right) \Leftrightarrow\left(R_{2} R_{0}\right)^{2 k} \in \operatorname{Stab}\left(H \varphi_{P}^{-1} g\right), \forall g \notin \Delta^{\hat{0}}, \\
& \left(R_{0} R_{1}\right)^{k} \in \operatorname{Stab}\left(H g \varphi_{P}\right) \Leftrightarrow\left(R_{0} R_{1}\right)^{2 k} \in \operatorname{Stab}\left(H \varphi_{P}^{-1} g\right), \forall g \in \Delta^{\hat{0}}, \\
& \left(R_{0} R_{1}\right)^{k} \in \operatorname{Stab}\left(H\left(g R_{0}\right) \varphi_{P}\right) \Leftrightarrow\left(R_{1} R_{0}\right)^{2 k} \in \operatorname{Stab}\left(H \varphi_{P}^{-1} g\right), \forall g \notin \Delta^{\hat{0}} .
\end{aligned}
$$

This clearly shows that $\operatorname{Pin}(\mathcal{H})$ is bipartite-uniform of bipartite-type $(1, l ; 2 m ; 2 n)$. Reciprocally, if $\operatorname{Pin}(\mathcal{H})$ is bipartite-uniform of bipartite-type $(1, l ; 2 m ; 2 n)$ then, reversing the above argument in a similar way as we did for $\operatorname{Wal}(\mathcal{H})$ in the proof of Theorem 10 , we easily conclude that $\mathcal{H}$ is uniform of type $(l ; m ; n)$.

(3) Since $\varphi_{P}$ is an epimorphism, $\mathcal{H}$ is regular $\Leftrightarrow H \triangleleft \Delta \Leftrightarrow H \varphi_{P}^{-1} \triangleleft \Delta^{\hat{0}} \Leftrightarrow \operatorname{Pin}(\mathcal{H})$ is bipartite-regular.

Theorem 13. If $\mathcal{H}$ is a bipartite hypermap such that all hypervertices in one $\Delta^{\hat{0}}$-orbit have valency 1 , then $\mathcal{H} \cong \operatorname{Pin}(\mathcal{G})$ for some hypermap $\mathcal{G}$.

Proof. As in Theorem 13, only the necessary condition needs to be proved. Let $H$ be a hypermap subgroup of $\mathcal{H}$. By taking $H^{R_{0}}$ instead of $H$ if necessary, we may assume, without loss of generality, that all hypervertices in the $\Delta^{\hat{0}}$-orbit of the hypervertex that contains the flag $H R_{0}$ have valency 1, i.e, $R_{1} R_{2} \in H^{R_{0} g}$ for all $g \in \Delta^{\hat{0}}$. Then $\left(\left(R_{1} R_{2}\right)^{R_{0}}\right)^{h} \in H$ for all $h \in \Delta^{\hat{0}}$; therefore $\operatorname{ker} \varphi_{P}=\left\langle\left(R_{1} R_{2}\right)^{R_{0}}\right\rangle^{\Delta^{\hat{0}}} \subseteq H$. This implies that $H \varphi_{P} \varphi_{P}^{-1}=H \operatorname{ker} \varphi_{P}=H$ and hence $\mathcal{H} \cong \operatorname{Pin}(\mathcal{G})$, where $\mathcal{G}$ is the hypermap with hypermap subgroup $G=H \varphi_{P}$.

Theorem 14. $\operatorname{Wal}\left(\mathcal{D}_{(01)}(\mathcal{H})\right) \cong \operatorname{Wal}(\mathcal{H})$.

Proof. If $H$ is a hypermap subgroup of $\mathcal{H}$, then $H \varphi_{W}^{-1}$ and $H(01)^{\circ} \varphi_{W}^{-1}$ are hypermap subgroups of $W a l(\mathcal{H})$ and $\operatorname{Wal}\left(\mathcal{D}_{(01)}(\mathcal{H})\right)$, respectively. Since $g \varphi_{W} \sigma=g \iota^{R_{0}} \varphi_{W}$ for all 
$g \in \Delta^{\hat{0}}$, where $\sigma=(01)^{\circ}$ and $\iota^{R_{0}}$ is the automorphism given by conjugation by $R_{0}$, we have

$$
H \sigma \varphi_{W}^{-1}=H \varphi_{W}^{-1} \iota^{R_{0}}
$$

that is, the hypermap subgroup $H(01)^{\circ} \varphi_{W}^{-1}$ of $\operatorname{Wal}\left(\mathcal{D}_{(01)}(\mathcal{H})\right)$ is just a conjugate under $R_{0}$ of the hypermap subgroup of $\operatorname{Wal}(\mathcal{H})$ and so they are isomorphic.

Theorem 15. $\operatorname{Pin}\left(\mathcal{D}_{(12)}(\mathcal{H})\right)=\mathcal{D}_{(12)}(\operatorname{Pin}(\mathcal{H}))$.

Proof. Let $H$ be a hypermap subgroup of $\mathcal{H}$ and $\sigma=(12)^{\circ}$. Then $H \sigma \varphi_{P}^{-1}$ and $H \varphi_{P}^{-1} \sigma$ are hypermap subgroups of $\operatorname{Pin}\left(\mathcal{D}_{(12)}(\mathcal{H})\right)$ and $\mathcal{D}_{(12)}(\operatorname{Pin}(\mathcal{H}))$, respectively. The equality $\sigma_{\left.\right|_{\Delta \hat{0}}} \varphi_{P}=\varphi_{P} \sigma$ actually shows that

$$
H \sigma \varphi_{P}^{-1}=H \varphi_{P}^{-1} \sigma
$$

so they represent the same hypermap.

Theorem 16. If $\operatorname{Wal}(\mathcal{H}) \cong \operatorname{Wal}(\mathcal{G})$, then $\mathcal{H} \cong \mathcal{G}$ or $\mathcal{H} \cong \mathcal{D}_{(01)}(\mathcal{G})$.

Proof. If $W a l(\mathcal{H}) \cong W a l(\mathcal{G})$ then $H \varphi_{W}^{-1}=\left(G \varphi_{W}^{-1}\right)^{g}$ for some $g \in \Delta$.

(i) $g \in \Delta^{\hat{0}}$. Then $\left(G \varphi_{W}^{-1}\right)^{g}=G^{g \varphi_{W}} \varphi_{W}^{-1}$, by Lemma $8(1)$, and then we have

$$
H=H \varphi_{W}^{-1} \varphi_{W}=G^{g \varphi_{W}} \varphi_{W}^{-1} \varphi_{W}=G^{g \varphi_{W}} ;
$$

that is, $\mathcal{H} \cong \mathcal{G}$.

(ii) $g \notin \Delta^{\hat{0}}$. Then $g R_{0} \in \Delta^{\hat{0}}$ and

$$
\left(G \varphi_{W}^{-1}\right)^{g}=\left(\left(G \varphi_{W}^{-1}\right)^{g R_{0}}\right)^{R_{0}}=\left(G^{\left(g R_{0}\right) \varphi_{W}} \varphi_{W}^{-1}\right)^{R_{0}}=G^{\left(g R_{0}\right) \varphi_{W}} \sigma \varphi_{W}^{-1},
$$

using (8), where $\lambda=\iota^{R_{0}}$ and $\sigma=(01)^{\circ}$. Therefore

$$
H=H \varphi_{W}^{-1} \varphi_{W}=G^{\left(g R_{0}\right) \varphi_{W}} \sigma \varphi_{W}^{-1} \varphi_{W}=G^{\left(g R_{0}\right) \varphi_{W}} \sigma,
$$

which says that $\mathcal{H} \cong D_{\sigma}(\mathcal{G})$.

Theorem 17. If $\operatorname{Pin}(\mathcal{H}) \cong \operatorname{Pin}(\mathcal{G})$, then $\mathcal{H} \cong \mathcal{G}$.

Proof. As before, let $H$ and $G$ be hypermap-subgroups of $\mathcal{H}$ and $\mathcal{G}$. If $\operatorname{Pin}(\mathcal{H}) \cong \operatorname{Pin}(\mathcal{G})$ then $H \varphi_{P}^{-1}=\left(G \varphi_{P}^{-1}\right)^{g}$ for some $g \in \Delta$.

(i) If $g \in \Delta^{\hat{0}}$ then, as before, $\left(G \varphi_{P}^{-1}\right)^{g}=G^{g \varphi_{P}} \varphi_{P}^{-1}$ and then $H=G^{g \varphi_{P}}$, showing that $\mathcal{H} \cong \mathcal{G}$.

(ii) Suppose that $g \notin \Delta^{\hat{0}}$. As for $b \in \Delta^{\hat{0}},\left(R_{1} R_{2}\right)^{R_{0} b} \varphi_{P}=1 \in H \cap G$ so that $\left(R_{1} R_{2}\right)^{R_{0} b}$ belongs to both $H \varphi_{P}^{-1}$ and $G \varphi_{P}^{-1}$, for all $b \in \Delta^{\hat{0}}$. Then $(1) R_{1} R_{2} \in\left(H \varphi_{P}^{-1}\right)^{b^{-1} R_{0}}$ and (2) since $\left(R_{1} R_{2}\right)^{R_{0} b g} \in\left(G \varphi_{P}^{-1}\right)^{g}=H \varphi_{P}^{-1}, \quad R_{1} R_{2} \in\left(H \varphi_{P}^{-1}\right)^{g^{-1} b^{-1} R_{0}}$. Since $b^{-1} R_{0}$ runs all over $\Delta \backslash \Delta^{\hat{0}}$ and $g^{-1} b^{-1} R_{0}$ runs all over $\Delta^{\hat{0}}$, when $b \in \Delta^{\hat{0}}$, then $R_{1} R_{2} \in\left(H \varphi_{P}^{-1}\right)^{d}$, for all $d \in \Delta$. This implies that all the hypervertices of $\operatorname{Pin}(\mathcal{H})$ have valency 1. By a dual version of Lemma $7, \operatorname{Pin}(\mathcal{H})$ is a "star"-like hypermap (see Figure 2); 


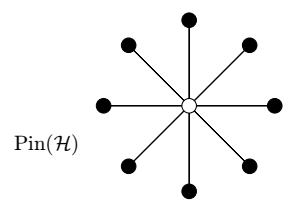

Figure 2: $\operatorname{Pin}(\mathcal{H})=D_{(02)}\left(\mathcal{D}_{n}\right)$.

that is, $\operatorname{Pin}(\mathcal{H})=D_{(02)}\left(\mathcal{D}_{n}\right)$. Hence $\operatorname{Pin}(\mathcal{H})$ is a regular hypermap on the sphere with $n$ (even) hypervertices. Thus $H \varphi_{P}^{-1}$, as well as $\left(G \varphi_{P}^{-1}\right)^{g}$, is normal in $\Delta$. Therefore, $H \varphi_{P}^{-1}=G \varphi_{P}^{-1}$ and hence $H=G$.

The proof of the above theorem reveals the following information,

Lemma 18. If Pin $(\mathcal{H})$ is not isomorphic to $D_{(02)}\left(\mathcal{D}_{n}\right)$ for any even $n$, then Pin $(\mathcal{H}) \cong$ $\operatorname{Pin}(\mathcal{G})$ implies that $H \varphi_{P}^{-1}=\left(G \varphi_{P}^{-1}\right)^{g}$ for some $g \in \Delta^{\hat{0}}$.

\subsection{Euler formula for bipartite-uniform hypermaps}

In this subsection we write the Euler characteristic of a bipartite-uniform hypermap in terms of its bipartite-type. Let $\mathcal{H}=\left(\Omega_{\mathcal{H}} ; h_{0}, h_{1}, h_{2}\right)$ be a bipartite-uniform hypermap with Euler characteristic $\chi$, let $V, E$ and $F$ be the numbers of hypervertices, hyperedges and hyperfaces of $\mathcal{H}$, respectively, and let $V_{1}$ and $V_{2}=V-V_{1}$ be the numbers of hypervertices of the two $\Delta^{\hat{0}}$-orbits in $\Omega_{\mathcal{H}}$. By Lemma 3, $\quad \chi=V_{1}+V_{2}+E+F-\frac{\left|\Omega_{\mathcal{H}}\right|}{2}$. Let $\left(l_{1}, l_{2} ; m ; n\right)$ be the bipartite-type of $\mathcal{H}$. Then $V_{1}=\frac{\left|\Omega_{\mathcal{H}}\right|}{4 l_{1}}, V_{2}=\frac{\left|\Omega_{\mathcal{H}}\right|}{4 l_{2}}, E=\frac{\left|\Omega_{\mathcal{H}}\right|}{2 m}$ and $F=\frac{\left|\Omega_{\mathcal{H}}\right|}{2 n}$. Replacing these values in the above formula we get the following result:

Lemma 19 (Euler formula for bipartite-uniform hypermaps). If $\mathcal{H}$ is a bipartiteuniform hypermap of bipartite-type $\left(l_{1}, l_{2} ; m ; n\right)$, then

$$
\chi=\frac{\left|\Omega_{\mathcal{H}}\right|}{2}\left(\frac{1}{2 l_{1}}+\frac{1}{2 l_{2}}+\frac{1}{m}+\frac{1}{n}-1\right) .
$$

\subsection{Spherical bipartite-uniform hypermaps}

In this subsection we classify the bipartite-uniform hypermaps $\mathcal{K}$ on the sphere. The main results were already given before; all we need now is to apply them directly to the sphere $(\chi=2)$.

Let $\mathcal{K}$ be a bipartite-uniform hypermap of bipartite-type $\left(l_{1}, l_{2} ; m ; n\right)$ on the sphere. Then $\chi=2>0$ and $\frac{1}{2 l_{1}}+\frac{1}{2 l_{2}}+\frac{1}{m}+\frac{1}{n}>1$. Suppose, without loss of generality, that $l_{1} \leq l_{2}$ and $m \leq n$. Then

$$
\begin{aligned}
\frac{1}{l_{1}}+\frac{2}{m} \geq \frac{1}{2 l_{1}}+\frac{1}{2 l_{2}}+\frac{1}{m}+\frac{1}{n}>1 & \Rightarrow \frac{1}{l_{1}}>\frac{1}{2} \quad \text { or } \quad \frac{2}{m}>\frac{1}{2} \\
& \Leftrightarrow l_{1}<2 \text { or } m<4 \\
& \Leftrightarrow l_{1}=1 \quad \text { or } m=2
\end{aligned}
$$

(since $m$ is even) 
From this result and Theorems 11 and 13, we deduce the following theorem.

Theorem 20. If $\mathcal{K}$ is a spherical bipartite-uniform hypermap, then $\mathcal{K} \cong W$ al $(\mathcal{R})$ or $\mathcal{K} \cong$ $\operatorname{Pin}(\mathcal{R})$ for some spherical uniform hypermap $\mathcal{R}$, unique up to isomorphism. Moreover, as $\mathcal{K}$ is bipartite-regular if and only if $\mathcal{R}$ is regular, and on the sphere all uniform hypermaps are regular, then all bipartite-uniform hypermaps on the sphere are bipartite-regular.

\begin{tabular}{|c|c|c|c|c|c|c|c|c|c|c|}
\hline$\#$ & $l_{1}$ & $l_{2}$ & $m$ & $n$ & $V_{1}$ & $V_{2}$ & $E$ & $F$ & $|\Omega|$ & $\mathcal{K}$ \\
\hline 1 & 1 & 1 & $2 n$ & $2 n$ & $n$ & $n$ & 1 & 1 & $4 n$ & $\operatorname{Pin}\left(\mathcal{D}_{(02)}\left(\mathcal{D}_{n}\right)\right)$ \\
\hline 2 & 1 & 2 & 4 & $2 n$ & $2 n$ & $n$ & $n$ & 2 & $8 n$ & $\operatorname{Pin}\left(\mathcal{P}_{n}\right)$ \\
\hline 3 & 1 & 2 & 6 & 6 & 12 & 6 & 4 & 4 & 48 & $\operatorname{Pin}\left(\mathcal{D}_{(01)}(\mathcal{T})\right)$ \\
\hline 4 & 1 & 2 & 6 & 8 & 24 & 12 & 8 & 6 & 96 & $\operatorname{Pin}\left(\mathcal{D}_{(01)}(\mathcal{C})\right)$ \\
\hline 5 & 1 & 2 & 6 & 10 & 60 & 30 & 20 & 12 & 240 & $\operatorname{Pin}\left(\mathcal{D}_{(01)}(\mathcal{D})\right)$ \\
\hline 6 & 1 & 3 & 4 & 6 & 12 & 4 & 6 & 4 & 48 & $\operatorname{Pin}(\mathcal{T})$ \\
\hline 7 & 1 & 3 & 4 & 8 & 24 & 8 & 12 & 6 & 96 & $\operatorname{Pin}(\mathcal{C})$ \\
\hline 8 & 1 & 3 & 4 & 10 & 60 & 20 & 30 & 12 & 240 & $\operatorname{Pin}(\mathcal{D})$ \\
\hline 9 & 1 & 4 & 4 & 6 & 24 & 6 & 12 & 8 & 96 & $\operatorname{Pin}\left(\mathcal{D}_{(02)}(\mathcal{C})\right)$ \\
\hline 10 & 1 & 5 & 4 & 6 & 60 & 12 & 30 & 20 & 240 & $\operatorname{Pin}\left(\mathcal{D}_{(02)}(\mathcal{D})\right)$ \\
\hline 11 & 1 & $n$ & 2 & $2 n$ & $n$ & 1 & $n$ & 1 & $4 n$ & $\operatorname{Pin}\left(\mathcal{D}_{(12)}\left(\mathcal{D}_{n}\right)\right)$ \\
\hline 12 & 1 & $n$ & 4 & 4 & $2 n$ & 2 & $n$ & $n$ & $8 n$ & $\operatorname{Pin}\left(\mathcal{D}_{(02)}\left(\mathcal{P}_{n}\right)\right)$ \\
\hline 13 & 2 & 2 & 2 & $2 n$ & $n$ & $n$ & $2 n$ & 2 & $8 n$ & $\operatorname{Wal}\left(\mathcal{P}_{n}\right)$ \\
\hline 14 & 2 & 3 & 2 & 6 & 6 & 4 & 12 & 4 & 48 & $W a l(\mathcal{T})$ \\
\hline 15 & 2 & 3 & 2 & 8 & 12 & 8 & 24 & 6 & 96 & Wal $(\mathcal{C})$ \\
\hline 16 & 2 & 3 & 2 & 10 & 30 & 20 & 60 & 12 & 240 & $\operatorname{Wal}(\mathcal{D})$ \\
\hline 17 & 2 & 4 & 2 & 6 & 12 & 6 & 24 & 8 & 96 & $\operatorname{Wal}\left(\mathcal{D}_{(02)}(\mathcal{C})\right)$ \\
\hline 18 & 2 & 5 & 2 & 6 & 30 & 12 & 60 & 20 & 240 & $W \operatorname{Wal}\left(\mathcal{D}_{(02)}(\mathcal{D})\right)$ \\
\hline 19 & 2 & $n$ & 2 & 4 & $n$ & 2 & $2 n$ & $n$ & $8 n$ & $W \operatorname{Wal}\left(\mathcal{D}_{(02)}\left(\mathcal{P}_{n}\right)\right)$ \\
\hline 20 & 3 & 3 & 2 & 4 & 4 & 4 & 12 & 6 & 48 & $W \operatorname{Wal}\left(\mathcal{D}_{(12)}(\mathcal{T})\right)$ \\
\hline 21 & 3 & 4 & 2 & 4 & 8 & 6 & 24 & 12 & 96 & $W \operatorname{Wal}\left(\mathcal{D}_{(12)}(\mathcal{C})\right)$ \\
\hline 22 & 3 & 5 & 2 & 4 & 20 & 12 & 60 & 30 & 240 & $\operatorname{Wal}\left(\mathcal{D}_{(12)}(\mathcal{D})\right)$ \\
\hline 23 & $n$ & $n$ & 2 & 2 & 1 & 1 & $n$ & $n$ & $4 n$ & $W a l\left(\mathcal{D}_{n}\right)$ \\
\hline
\end{tabular}

Table 2: The bipartite-regular hypermaps on the sphere.

Based on the knowledge of regular hypermaps on the sphere, we display in Table 2 all the possible values (up to duality) for the bipartite-type of the bipartite-regular hypermaps on the sphere and the unique hypermap (up to isomorphism) with such a bipartite-type. Notice that the map of bipartite-type $(1, n ; 2 ; 2 n)$ can be constructed from $\mathcal{D}_{n}$ either via a Wal transformation $\operatorname{Wal}\left(\mathcal{D}_{(02)}\left(\mathcal{D}_{n}\right)\right)$ or via a Pin transformation $\operatorname{Pin}\left(\mathcal{D}_{(12)}\left(\mathcal{D}_{n}\right)\right)$. Since $\operatorname{Wal}\left(\mathcal{D}_{(02)}\left(\mathcal{D}_{n}\right)\right) \cong \operatorname{Wal}\left(\mathcal{D}_{(12)}\left(\mathcal{D}_{n}\right)\right)$ these two constructions (Wal and Pin) can actually be carried forward on the same hypermap $\mathcal{D}_{(12)}\left(\mathcal{D}_{n}\right)$. The Tetrahedron $\mathcal{R}=\mathcal{T}$, which is self-dual, gives rise to $\operatorname{Wal}\left(\mathcal{D}_{(01)}(\mathcal{T})\right)=W a l(\mathcal{T})=\operatorname{Wal}\left(\mathcal{D}_{(02)}(\mathcal{T})\right)$.

\section{$3 \quad$ Irregularity and chirality}

We follow the same terminology and notations used in [3]. Let $\mathcal{K}$ be a bipartite (that is, $\Delta^{\hat{0}}$-conservative) hypermap with hypermap-subgroup $K<\Delta^{\hat{0}}$. If $\mathcal{K}$ is not regular (that is, not $\Delta$-regular), then its closure cover $\mathcal{K}^{\Delta}$ is the largest regular hypermap covered by $\mathcal{K}$ and its covering core $\mathcal{K}_{\Delta}$ is the smallest regular hypermap covering $\mathcal{K}$. Hence we have two 
normal subgroups in $\Delta$, the normal closure $K^{\Delta}$ containing $K$, and the core $K_{\Delta}$ contained in $K$. Since $K_{\Delta} \triangleleft K$, although $K$ may not be normal in $K^{\Delta}$, we have a group

$$
\Upsilon_{\Delta}(\mathcal{K})=K / K_{\Delta}
$$

called the lower-irregularity group of $\mathcal{K}$. Its size is the lower-irregularity index and is denoted by $\iota_{\Delta}(\mathcal{K})$. The upper-irregularity index, denoted by $\iota^{\Delta}(\mathcal{K})$, is the index $\left|K^{\Delta}: K\right|$. If $\mathcal{K}$ is bipartite-regular, then $K \triangleleft \Delta^{\hat{0}}$, and since $K^{\Delta}$ is a subgroup of $\Delta^{\hat{0}}, K \triangleleft K^{\Delta}$ and we have another group, the upper-irregularity group

$$
\Upsilon^{\Delta}(\mathcal{K})=K^{\Delta} / K
$$

Since the index of $\Delta^{\hat{0}}$ in $\Delta$ is 2 , the upper- and lower-irregularity groups are isomorphic; so their upper- and lower-irregularity indices are equal $(\mathcal{K}$ is irregularity balanced). The common group $\Upsilon^{\Delta}(\mathcal{K}) \cong \Upsilon_{\Delta}(\mathcal{K})=\Upsilon$ is the irregularity group of the bipartite-regular hypermap $\mathcal{K}$ and the common value $\iota^{\Delta}(\mathcal{K})=\iota_{\Delta}(\mathcal{K})=\iota$ is its irregularity index. This has value 1 if and only if $\mathcal{K}$ is regular. Being bipartite-regular, $\mathcal{K}$ is isomorphic to a regular $\Delta^{\hat{0}}$-marked hypermap (see $[1]$ )

$$
\mathcal{Q}=(G, a, b, c, d) \cong\left(\Delta^{\hat{0}} / K, K A, K B, K C, K D\right),
$$

where $\Delta^{\hat{0}}=\langle A, B, C, D\rangle \cong C_{2} * C_{2} * C_{2} * C_{2}$ and $K$ is the $\Delta^{\hat{0}}$-hypermap subgroup of $\mathcal{Q}$ (and the hypermap subgroup of $\mathcal{K}$ ). Here $G$ is the group generated by $a, b, c, d$. To compute the irregularity group of $\mathcal{K}$ we use:

Lemma 21. If $G$ has presentation $\langle a, b, c, d \mid R=1\rangle$, where $R=\left\{R_{1}, \ldots, R_{k}\right\}$ is a set of relators $R_{i}=R_{i}(a, b, c, d)$ then $\Upsilon^{\Delta}(\mathcal{K})=\left\langle R^{R_{0}}\right\rangle^{G}$.

See [3] for the proof.

The definition of chirality given in [2] is slightly different from that used in $[6,7,8,9]$. If $\mathcal{K}$ is bipartite $\left(K<\Delta^{\hat{0}}\right)$, not necessarily bipartite-regular, then $\mathcal{K}$ is $\Delta^{\hat{0}}$-chiral, or bipartite-chiral, if the normaliser $N_{\Delta}(K)$ of $K$ in $\Delta$ is a subgroup of $\Delta^{\hat{0}}$. In other words, $\mathcal{K}$ is $\Delta^{0}$-chiral if the group of automorphisms $A u t(\mathcal{K}) \cong N_{\Delta}(K) / K$ contains no "symmetry" besides $\Delta^{\hat{0}}$.

Let $\mathcal{K}$ be a $\Delta^{\hat{0}}$-chiral hypermap. If $\mathcal{K}$ is bipartite-regular $\left(\Delta^{\hat{0}}\right.$-regular), then $K \triangleleft \Delta^{\hat{0}}$ and so we have $N_{\Delta}(K)=\Delta^{\hat{0}}$. Thus $\mathcal{K}$ is $\Delta^{\hat{0}}$-chiral if and only if $K$ is not normal in $\Delta$; that is, if and only if $\mathcal{K}$ is irregular. As $\Delta^{\hat{0}}$ has index 2 in $\Delta$, with transversal $\left\{1, R_{0}\right\}$, we have $K=K^{\left\langle R_{0}\right\rangle}=K K^{R_{0}}=K^{\Delta}$ if and only if $R_{0} \in N_{\Delta}(K)$; that is, if and only if $K R_{0} \in \operatorname{Aut}(\mathcal{K})$. Hence the upper-irregularity index $\iota^{\Delta}$ gives a "measure" of "how close" $\mathcal{K}$ is to having the "symmetry" $K R_{0}$ outside $\Delta^{\hat{0}}$. For this reason we also call the upper-irregularity index (which coincides with the lower-irregularity index) the $\Delta^{\hat{0}}$ chirality index of the bipartite-regular $\mathcal{K}$. This expresses how "close" $\mathcal{K}$ is to getting a "symmetry" outside $\Delta^{\hat{0}}$, or in other words, how close it is to losing $\Delta^{\hat{0}}$-chirality.

The same happens to any normal subgroup $\Theta$ with index two in $\Delta$. In particular, for $\Theta=\Delta^{+}$, the upper irregularity index (or simply the irregularity index) of a $\Delta^{+}$-regular 
(that is, orientably regular) hypermap coincides with the $\Delta^{+}$-chirality index. This explains the use of chirality index in place of irregularity index (of orientably regular hypermaps) in the papers $[6,7,8,9]$. For more information and a general definition of chirality group see $[2]$.

\begin{tabular}{|c|c|c|c|c|c|c|c|c|c|}
\hline $\mathcal{K}$ & $\mathcal{K}^{\Delta}$ & $l, m, n$ & $|\Omega|$ & $\mathcal{K}_{\Delta}$ & $l, m, n$ & $|\Omega|$ & genus & $\iota$ & $\Upsilon$ \\
\hline $\operatorname{Pin}\left(\mathcal{D}_{(02)}\left(\mathcal{D}_{n}\right)\right)$ & $\mathcal{D}_{(02)}\left(\mathcal{D}_{2 n}\right)$ & $1,2 n, 2 n$ & $4 n$ & $\mathcal{D}_{(02)}\left(\mathcal{D}_{2 n}\right)$ & $1,2 n, 2 n$ & $4 n$ & 0 & 1 & 1 \\
\hline & $\left\{\mathcal{D}_{(02)}\left(\mathcal{D}_{4}\right)\right.$ & $1,4,4$ & 8 & $\mathcal{K}_{\Delta 2}$ & $2,4,2 n$ & $8 n^{2}$ & $\frac{(n-1)^{2}+1}{2}$ & $n$ & $D_{\frac{n}{2}}, n$ even \\
\hline$P \imath n\left(P_{n}\right)$ & $\left\{\mathcal{D}_{(02)}\left(\mathcal{D}_{2}\right)\right.$ & $1,2,2$ & 4 & $\mathcal{K}_{\Delta 3}$ & $2,4,2 n$ & $16 n^{2}$ & $(n-1)^{2}$ & $2 n$ & $D_{n}^{2}, n$ odd \\
\hline $\operatorname{Pin}\left(\mathcal{D}_{(01)}(\mathcal{T})\right)$ & $\mathcal{D}_{(02)}\left(\mathcal{D}_{6}\right)$ & $1,6,6$ & 12 & $\mathcal{K}_{\Delta_{4}}$ & $2,6,6$ & 192 & 9 & 4 & $V_{4}$ \\
\hline $\operatorname{Pin}\left(\mathcal{D}_{(01)}(\mathcal{C})\right)$ & $\mathcal{D}_{(02)}\left(\mathcal{D}_{2}\right)$ & $1,2,2$ & 4 & $\mathcal{K}_{\Delta 5}$ & $2,6,8$ & 2304 & 121 & 24 & $S_{4}$ \\
\hline $\operatorname{Pin}\left(\mathcal{D}_{(01)}(\mathcal{D})\right)$ & $\mathcal{D}_{(02)}\left(\mathcal{D}_{2}\right)$ & $1,2,2$ & 4 & $\mathcal{K}_{\Delta 6}$ & $2,6,10$ & 14400 & 841 & 60 & $A_{5}$ \\
\hline $\operatorname{Pin}(\mathcal{T})$ & $\mathcal{D}_{(02)}\left(\mathcal{D}_{2}\right)$ & $1,2,2$ & 4 & $\mathcal{K}_{\Delta 7}$ & $3,4,6$ & 576 & 37 & 12 & $A_{4}$ \\
\hline $\operatorname{Pin}(\mathcal{C})$ & $\mathcal{D}_{(02)}\left(\mathcal{D}_{4}\right)$ & $1,4,4$ & 8 & $\mathcal{K}_{\Delta 8}$ & $3,4,8$ & 1152 & 85 & 12 & $A_{4}$ \\
\hline $\operatorname{Pin}(\mathcal{D})$ & $\mathcal{D}_{(02)}\left(\mathcal{D}_{2}\right)$ & $1,2,2$ & 4 & $\mathcal{K}_{\Delta 9}$ & $3,4,10$ & 14400 & 1141 & 60 & $A_{5}$ \\
\hline $\operatorname{Pin}\left(\mathcal{D}_{(02)}(\mathcal{C})\right)$ & $\mathcal{D}_{(02)}\left(\mathcal{D}_{2}\right)$ & $1,2,2$ & 4 & $\mathcal{K}_{\Delta 10}$ & $4,4,6$ & 2304 & 193 & 24 & $S_{4}$ \\
\hline $\operatorname{Pin}\left(\mathcal{D}_{(02)}(\mathcal{D})\right)$ & $\mathcal{D}_{(02)}\left(\mathcal{D}_{2}\right)$ & $1,2,2$ & 4 & $\mathcal{K}_{\Delta 11}$ & $5,4,6$ & 14400 & 1381 & 60 & $A_{5}$ \\
\hline $\operatorname{Pin}\left(\mathcal{D}_{(12)}\left(\mathcal{D}_{n}\right)\right)$ & $\mathcal{D}_{(02)}\left(\mathcal{D}_{2}\right)$ & $1,2,2$ & 4 & $\mathcal{K}_{\Delta 12}$ & $n, 2,2 n$ & $4 n^{2}$ & $\frac{(n-1)(n-2)}{2}$ & $n$ & $C_{n}$ \\
\hline $\operatorname{Pin}\left(\mathcal{D}_{(02)}\left(\mathcal{P}_{n}\right)\right)$ & $\mathcal{D}_{(02)}\left(\mathcal{D}_{4}\right)$ & $1,4,4$ & 8 & $\mathcal{K}_{\Delta 13}$ & $n, 4,4$ & $8 n^{2}$ & $(n-1)^{2}$ & $n$ & $C_{n}$ \\
\hline $\operatorname{Wal}\left(\mathcal{P}_{n}\right)$ & $\mathcal{P}_{2 n}$ & $2,2,2 n$ & $8 n$ & $\mathcal{P}_{2 n}$ & $2,2,2 n$ & $8 n$ & 0 & 1 & 1 \\
\hline$W a l(\mathcal{T})$ & $\mathcal{D}_{(02)}\left(\mathcal{D}_{2}\right)$ & $1,2,2$ & 4 & $\mathcal{K}_{\Delta 15}$ & $6,2,6$ & 576 & 25 & 12 & $A_{4}$ \\
\hline $\operatorname{Wal}(\mathcal{C})$ & $\mathcal{D}_{(02)}\left(\mathcal{D}_{2}\right)$ & $1,2,2$ & 4 & $\mathcal{K}_{\Delta 16}$ & $6,2,8$ & 2304 & 121 & 24 & $S_{4}$ \\
\hline $\operatorname{Wal}(\mathcal{D})$ & $\mathcal{D}_{(02)}\left(\mathcal{D}_{2}\right)$ & $1,2,2$ & 4 & $\mathcal{K}_{\Delta 17}$ & $6,2,10$ & 14400 & 841 & 60 & $A_{5}$ \\
\hline$W a l\left(\mathcal{D}_{(02)}(\mathcal{C})\right)$ & $\mathcal{P}_{6}$ & $2,2,6$ & 24 & $\mathcal{K}_{\Delta 18}$ & $4,2,6$ & 384 & 9 & 4 & $V_{4}$ \\
\hline$W a l\left(\mathcal{D}_{(02)}(\mathcal{D})\right)$ & $\mathcal{D}_{(02)}\left(\mathcal{D}_{2}\right)$ & $1,2,2$ & 4 & $\mathcal{K}_{\Delta 19}$ & $10,2,6$ & 14400 & 841 & 60 & $A_{5}$ \\
\hline$W a l\left(\mathcal{D}_{(0}\right.$ & $\left\{\mathcal{P}_{4}\right.$ & $2,2,4$ & 16 & $\mathcal{K}_{\Delta 20}$ & & $4 n^{2}$ & $\frac{(n-2)^{2}}{4}$ & $\frac{n}{2}$ & $C_{\frac{n}{2}}, n$ even \\
\hline (02) & $\left(\mathcal{D}_{(02)}\left(\mathcal{D}_{2}\right)\right.$ & $1,2,2$ & 4 & $\mathcal{K}_{\Delta 21}$ & $2 n, 2,4$ & $16 n^{2}$ & & $2 n$ & $D_{n}, n$ odd \\
\hline$W a l\left(\mathcal{D}_{(12)}(\mathcal{T})\right)$ & $\mathcal{C}$ & $3,2,4$ & 48 & $\mathcal{C}$ & $3,2,4$ & 48 & 0 & 1 & 1 \\
\hline$W a l\left(\mathcal{D}_{(12)}(\mathcal{C})\right)$ & $\mathcal{D}_{(02)}\left(\mathcal{D}_{2}\right)$ & $1,2,2$ & 4 & $\mathcal{K}_{\Delta 23}$ & $12,2,4$ & 2304 & 97 & 24 & $S_{4}$ \\
\hline$W a l\left(\mathcal{D}_{(12)}(\mathcal{D})\right)$ & $\mathcal{D}_{(02)}\left(\mathcal{D}_{2}\right)$ & $1,2,2$ & 4 & $\mathcal{K}_{\Delta 24}$ & $15,2,4$ & 14400 & 661 & 60 & $A_{5}$ \\
\hline$W a l\left(\mathcal{D}_{n}\right)$ & $\mathcal{D}_{(02)}\left(\mathcal{P}_{n}\right)$ & $n, 2,2$ & $4 n$ & $\mathcal{D}_{(02)}\left(\mathcal{P}_{n}\right)$ & $n, 2,2$ & $4 n$ & 0 & 1 & 1 \\
\hline
\end{tabular}

Table $3: \mathcal{K}, \mathcal{K}^{\Delta}$ and $\mathcal{K}_{\Delta}$

\section{Computing the irregularity group $\Upsilon$.}

Let $\mathcal{K}=\mathcal{H}^{\varphi^{-1}}=\operatorname{Wal}(\mathcal{H})$ or $\operatorname{Pin}(\mathcal{H})$ conform $\varphi=\varphi_{W}$ or $\varphi_{P}$, respectively, and let $H$ be the hypermap subgroup of a regular hypermap $\mathcal{H}$ of type $(l ; m ; n)$. The inverse image $K=H \varphi^{-1}$ is the hypermap subgroup of $\mathcal{K}$. The lower-irregularity index of $\mathcal{K}, \Upsilon_{\Delta}(\mathcal{K})=$ $K / K_{\Delta}$, is isomorphic to its upper-irregularity group $\Upsilon^{\Delta}(\mathcal{K})=K^{\Delta} / K$, a subgroup of the $\Delta^{\hat{0}}$-monodromy group $G=\Delta^{\hat{0}} / K$ of $\mathcal{K}$. This common group, the irregularity group $\Upsilon$, can be computed in the following way. According to Theorem 9 , the group $G \cong \operatorname{Mon}(\mathcal{H})$ is a known group (see Table 1). Being $G$ the $\Delta^{\hat{0}}$-monodromy group of a bipartite-regular hypermap, using $\varphi$ we can rewrite $G$ in the following form

$$
G=\left\langle a, b, c, d \mid a^{2}=b^{2}=c^{2}=d^{2}=1, R=1\right\rangle,
$$

such that $a^{R_{0}}=c, b^{R_{0}}=d, c^{R_{0}}=a$ and $d^{R_{0}}=b ; \quad R$ stands for a set of relators on $a, b, c, d$. By Lemma 21,

$$
\Upsilon=\left\langle R^{R_{0}}\right\rangle^{G}
$$


is the closure subgroup of $R^{R_{0}}$ in $G$. This calculation is easily performed and the results for $\Upsilon(\mathcal{K})$ can be seen in the last column of Table 3. For an example of how this calculation is carried out see Theorem 23.

However, since $\varphi=\varphi_{W}$ or $\varphi_{P}$ sends generators of $\Delta^{\hat{0}}$ of odd length in $\Delta$ to generators of $\Delta$ of odd length in $\Delta$, we have necessarily $\Delta^{+} \varphi^{-1}=\Delta^{+\hat{0}}$, where $\Delta^{+\hat{0}}=\Delta^{+} \cap \Delta^{\hat{0}}$. Since $H \triangleleft \Delta^{+}$, then $K^{\Delta} / K \triangleleft \Delta^{+\hat{0}} / K=\Delta^{+} \varphi^{-1} / H \varphi^{-1} \cong \Delta^{+} / H=A u t^{+}(\mathcal{H})$; that is, $\Upsilon=K^{\Delta} / K$ is a normal subgroup of $A u t^{+}(\mathcal{H})$.

Let $A=R_{1}, B=R_{2}, C=R_{1}^{R_{0}}$ and $D=R_{2}^{R_{0}}$. Then $\Delta^{\hat{0}}=\langle A, B, C, D\rangle$. (1) If $\mathcal{K}=\operatorname{Wal}(\mathcal{H})$ then $K=\left\langle B D,(A B)^{l},(D C)^{m},(C A)^{n}\right\rangle^{\Delta^{\hat{0}}}$ so $K^{\Delta}=\left\langle B D,(A B)^{l}\right.$, $\left.(D C)^{m},(C A)^{n}\right\rangle^{\Delta}$. Let $d=\operatorname{gcd}(l, m)$. Since $(A B)^{m}=\left((D C)^{-m}\right)^{R_{0}}$ and $(D C)^{l}=\left((A B)^{-l}\right)^{R_{0}}$ then $(A B)^{d}$ and $(D C)^{d}$ also belong to $K^{\Delta}$. Hence if $d=1$, then $A B$ and $D C$ belong to $K^{\Delta}$ and so $K^{\Delta}=\Delta^{+0}$. Therefore $\Upsilon=A u t^{+}(\mathcal{H})$ when $d=1$.

(2) If $\mathcal{K}=\operatorname{Pin}(\mathcal{H})$, then $K=\left\langle C D,(A B)^{l},(B D)^{m},(C A)^{n}\right\rangle^{\Delta^{0}} ;$ so $K^{\Delta}=\left\langle C D,(A B)^{l}\right.$, $\left.(B D)^{m},(C A)^{n}\right\rangle^{\Delta}$. Let $d=\operatorname{gcd}(m, n)$. Since $K^{\Delta} D=K^{\Delta} C, K^{\Delta}(C A)^{m}=K^{\Delta}(D A)^{m}=$ $\left(K^{\Delta}(B C)^{m}\right)^{R_{0}}=\left(K^{\Delta}(B D)^{m}\right)^{R_{0}}=K^{\Delta}$ and so $(C A)^{m} \in K^{\Delta}$. Similarly, $(B D)^{n} \in K^{\Delta}$. Hence if $d=1$, then $K^{\Delta}=\Delta^{+0}$ and consequently $\Upsilon=A u t^{+}(\mathcal{H})$.

Therefore the general calculations mentioned above only need to be carried out for the cases where $d \neq 1$, namely the cases 2 (for $n$ even), 3, 7, 12, 17 and 19 (for $n$ even).

\section{Computing the closure cover $\mathcal{K}^{\Delta}$.}

Once the irregularity index is calculated, it is an easy task to compute the closure cover $\mathcal{K}^{\Delta}$ of $\mathcal{K}=\mathcal{H}^{\varphi^{-1}}$, simply because the genus of the closure cover is zero and in the sphere the type determines uniquely a uniform (or regular) hypermap. Let $(l ; m ; n)$ be the type of the closure cover $\mathcal{K}^{\Delta}$ and let $(r, s ; u ; v)$ be the bipartite-type of the spherical bipartiteregular hypermap $\mathcal{K}$. The number of flags $\left|\Omega_{\mathcal{K}^{\Delta}}\right|$ of the closure cover must divide the number of flags $\left|\Omega_{\mathcal{K}}\right|$ of $\mathcal{K}$. Also $l$ divides $\operatorname{gcd}(r, s), m$ divides $u$ and $n$ divides $v$. The greatest possible values for $l, m$ and $n$ are $\operatorname{gcd}(r, s), u$ and $v$, respectively. Moreover, when $\operatorname{gcd}(r, s)=1$ we must have $l=1$ in which case $m=n$ and the greatest possible values are achieved for $m=n=\operatorname{gcd}(u, v)$. Since $\mathcal{K}^{\Delta}$ is a regular hypermap on the sphere and is determined by $l, m$ and $n$, we must check if in each case the above choice of $l, m$ and $n$ give rise to a spherical type (cf. Table 1). If not we choose the second greatest, the third greatest and so forth. For each bipartite-regular hypermap $\mathcal{K}$ in Table 2, where $\left(l_{1}, l_{2} ; m ; n\right)$ is our $(r, s ; u ; v)$, taking the greatest values for the triple $(l, m, n)$ we get a spherical type. To check if such triple determines a hypermap covered by $\mathcal{K}$ we take a half-turn in the middle of each hyperedge of $\mathcal{K}$; these half-turns determine a covering $\mathcal{K} \mapsto \mathcal{K}^{\Delta}$. The results can be seen in Table 3 .

\section{Computing the covering core $\mathcal{K}_{\Delta}$.}

The covering core is already computed since we know its monodromy group

$$
\operatorname{Mon}\left(\mathcal{K}_{\Delta}\right)=\operatorname{Mon}(\mathcal{K})
$$


and their canonical generators. Feeding these parameters in GAP [12], for example, we get the rest of the information shown in the Table 3 . In this table we observe two isolated maps (not in families) with less then 100 edges, the map $D_{(12)}\left(\mathcal{K}_{\Delta 4}\right)$ with 48 edges and Petrie path of length 4 , and $\mathcal{K}_{\Delta 18}$ with 96 edges and Petrie path of length 6 . In [25], where we can find a good list of regular maps up to 100 edges (although the list is guaranteed to be complete only up to 49), these maps are $P(70)$ and $D P(190)$ on pages 144 and 181 respectively. These can be consulted in the recently created Census of orientably-regular maps [27].

\section{Final comments}

By examining Table 3 we observe the following extra result,

Theorem 22. The irregularity (or chirality) index of a bipartite-regular hypermap can be any positive integer number. Moreover, cyclic groups and dihedral groups are irregularity groups of bipartite-regular hypermaps.

Using the Pin and Wal transformations we can say a little more.

Theorem 23. On each orientable surface of genus $g$ there are $\Delta^{\hat{0}}$-chiral hypermaps (that is, irregular bipartite-regular hypermaps) with irregularity indices $2 g+1,4 g+2$ and $4 g$.

Proof. Just take the $\operatorname{Pin}\left(\mathcal{M}_{k}\right)$ and the $\operatorname{Wal}\left(\mathcal{M}_{k}\right)$ constructions over the one-face regular map $\mathcal{M}_{k}$ formed from a single $2 k$-gon by identifying opposite edges orientably. The map $\mathcal{M}_{k}$ has type $(k ; 2 ; 2 k)$ or $(2 k ; 2 ; 2 k)$ according as $k$ is odd or even. The monodromy group of $\mathcal{M}_{k}$ is the dihedral group $D_{2 k}$ generated by the involutions $r_{0}, r_{1}$ and $r_{2}$ subject to the relations $\left(r_{0} r_{1}\right)^{2 k}=1$ and $r_{2}=r_{0}\left(r_{1} r_{0}\right)^{k}$. The genus of $\mathcal{M}_{k}$ is $\frac{k-1}{2}$ if $k$ is odd and $\frac{k}{2}$ otherwise. Hence each orientable surface of genus $g$ supports two maps $\mathcal{M}_{k}$, one for $k$ odd and another for $k$ even. Note that $\mathcal{M}_{k}$ has 1 or 2 vertices according as $k$ is even or odd.

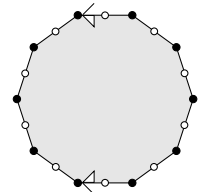

(a)

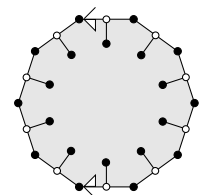

(b)

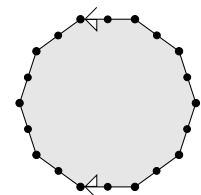

(c)

Figure 3: (a) The $\mathcal{M}_{k}$ map (opposite edges identified orientably).

(b) $\operatorname{Pin}\left(\mathcal{M}_{k}\right)$. (c) $\operatorname{Wal}\left(\mathcal{M}_{k}\right)$.

The bipartite-regular hypermap $\operatorname{Pin}\left(\mathcal{M}_{k}\right)$ has bipartite-type $(1, k ; 4 ; 4 k)$ if $k$ is odd and $(1,2 k ; 4 ; 4 k)$ otherwise. The bipartite-regular map $\operatorname{Wal}\left(\mathcal{M}_{k}\right)$ has type $(2, k ; 2 ; 4 k)$ or $(2,2 k ; 2 ; 4 k)$ according as $k$ is odd or even. Let $H$ be the hypermap subgroup of $\mathcal{M}_{k}$ and $K=H \varphi^{-1}$, where $\varphi=\varphi_{P}$ or $\varphi_{W}$.

(1) The hypermap $\operatorname{Pin}\left(\mathcal{M}_{k}\right)$. The epimorphism $\varphi_{P}$ induces an isomorphism $G=\Delta^{\hat{0}} / K \rightarrow$ $\Delta / H$, mapping $a \mapsto r_{1}, b \mapsto r_{2}, c \mapsto r_{0}$ and $d \mapsto r_{0}$. That is, $c=d$ in the $\Delta^{\hat{0}}$-monodromy 
group of $\operatorname{Pin}\left(\mathcal{M}_{k}\right)$. With the help of $\varphi_{P}$ we rewrite $\operatorname{Mon}\left(\mathcal{M}_{k}\right)$ in function of $a, b, c$ and $d$ to get the $\Delta^{\hat{0}}$-monodromy group

$$
G=\left\langle a, b, c, d \mid a^{2}=b^{2}=c^{2}=d^{2}=1, c=d,(c a)^{2 k}=1, b=c(a c)^{k}\right\rangle .
$$

In this case $R=\left\{c d^{-1},(a c)^{2 k}, c(a c)^{k} b^{-1}\right\}$ and the irregularity group of $\operatorname{Pin}\left(\mathcal{M}_{k}\right)$ is the normal closure of $R^{R_{0}}$ in $G$; thus

$$
\Upsilon=\left\langle a b^{-1},(c a)^{2 k}, a(c a)^{k} d^{-1}\right\rangle^{G}=\langle a b\rangle^{G}=\langle a b\rangle .
$$

Since $a b=(a c)^{k+1}$, this group has size $k$ if $k$ is odd and size $2 k$ otherwise. Hence $\operatorname{Pin}\left(\mathcal{M}_{k}\right)$ has irregularity index $\iota=k=2 g+1$, for $k$ odd, and $\iota=2 k=4 g$ for $k$ even.

(2) The map $W a l\left(\mathcal{M}_{k}\right)$. Proceeding similarly we obtain

$$
\begin{aligned}
G & =\Delta^{\hat{0}}-\operatorname{Mon}\left(\operatorname{Wal}\left(\mathcal{M}_{k}\right)\right) \\
& =\left\langle a, b, c, d \mid a^{2}=b^{2}=c^{2}=d^{2}=1, b=d,(c a)^{2 k}=1, b=c(a c)^{k}\right\rangle
\end{aligned}
$$

and irregularity group $\Upsilon=\langle a c\rangle=C_{2 k}$ cyclic, giving rise to irregularity indices $\iota=2 k=$ $4 g+2$ when $k$ is odd and $\iota=2 k=4 g$ when $k$ is even.

For non-orientable surfaces we cannot answer affirmatively since to obtain $\Delta^{\hat{0}}$-chiral hypermaps the Pin and Wal constructions need regular hypermaps and we know that there are none on the non-orientable surfaces with negative characteristic $0,1,16,22,25$, 37 , and $46[5,28]$.

\section{References}

[1] A. Breda d'Azevedo, A theory of restricted regularity of hypermaps, J. Korean Math. Soc., 43 (2006), n $\mathrm{n}^{o}$ 5, 991-1018.

[2] - Restricted chirality of hypermaps, submitted.

[3] A. Breda d'Azevedo, D. Catalano, R. Duarte, Irregularity of restrictedly regular hypermaps, submitted.

[4] A. Breda d'Azevedo, G. Jones, Double coverings and reflexible abelian hypermaps, Beitr. Algebra Geom., 41 (2000), n 2, 371-389.

[5] - Rotary hypermaps of genus 2, Beitr. Algebra Geom., 42 (2001), nº 1, 39-58.

[6] A. Breda d'Azevedo, G. Jones, R. Nedela and M. Škoviera, Chirality groups of maps and hypermaps, due to appear in the Journal of Algebraic Combinatorics.

[7] A. Breda d'Azevedo, R. Nedela, Chiral hypermaps of small genus, Beitr. Algebra Geom., 44 (2003), no 1, 127-143.

[8] - Chiral hypermaps with few hyperfaces, Math. Slovaca, 53 (2003), n ${ }^{o}$ 2, $107-128$. 
[9] - Join and Intersection of hypermaps, Acta Univ. M. Belii Math., 9 (2001) $13-28$.

[10] H.S.M. Coxeter, W.O.J. Moser, "Generators and relations for discrete groups", Springer-Verlag, New York, 1984, 4th edition.

[11] W. Dyck, Über Ausfstellung und Untersuchung von Gruppe und Irrationalität regulären Riemannscher Flächen, Math. Ann., 17 (1880) 473-508.

[12] The GAP Group, GAP - Groups, Algorithms, and Programming, Version 4.4; 2004, (http://www.gap-system.org).

[13] F. Klein, Über die Transformation siebenter Ordnung der elliotischen Funktionen, Math. Ann., 14 (1879) 428-471.

[14] L.D. James, Operations on hypermaps and outer automorphism, European J. Combinatorics, 9 (1988) 551-560.

[15] L.D. James, G. A. Jones, Regular orientable imbeddings of complete graphs, J. Combin. Theory Ser. B, 39 (1985) 353-367.

[16] D.L. Johnson, "Topics in the Theory of Groups Presentations", London Math. Soc. Lecture Note Series 42, Cambridge University Press, 1980.

[17] J.E. Graver, M.E. Watkins, Locally finite, planar, edge-transitive graphs, Memoirs Am Math Soc, 126 (601)(1997).

[18] G.A. Jones, Just-edges-transitive maps and Coxeter groups, Ars Comb, 16 (1983) $139-150$.

[19] - Operations on maps and hypermaps, 13th Séminaire Lotharingien de Combinatoire, at Castel San Pietro Terme, Bologna, September 1985.

[20] G.A. Jones, J.S. Thornton, Operations on maps, and outer automorphisms, J. Combin. Theory Ser. B, 35 (1983) 93-103.

[21] C. H. Sah, Groups related to compact Riemann surfaces, Acta Math., 123 (1969) $13-42$.

[22] J. Širan,T.W. Tucker and M.E. Watkins, Realizing finite edge-transitive orientable maps, J. Graph Theory, 37 (2001) 1-34.

[23] W. Thomson, "The Robert Boyle Lecture, Oxford University Junior Scientific Club", May 16, 1893, reprinted in Baltimore Lectures (C. J. Clay \& Sons, London,1904).

[24] T.R.S. Walsh, Hypermaps versus bipartite maps, J. Combinatorial Theory, 18 (1975), $\mathrm{n}^{\circ} \mathrm{B}, 155-163$.

[25] S.E. Wilson, New Techniques for the Construction of Regular Maps, Ph.D. Thesis, University of Washington, 1976.

[26] - Bicontactual regular maps, Pacific J. Math., 2 (1985), no 120, 437-451.

[27] — Census of rotary maps, http://www.ijp.si/RegularMaps/.

[28] S. Wilson, A. Breda d'Azevedo, Surfaces with no regular hypermaps, Discrete Math., 277 (2004) 241-274. 